\title{
Técnicas de control para el balance de un robot bípedo: un estado del arte
}

\section{Control techniques for the balance of a robot biped: a state of the art}

\author{
Sergey GonZález MeJía \\ Ingeniero electrónico, especialista en Automatización Industrial, máster en \\ Ingeniería-énfasis en Automática, investigador de la Universidad del Valle, \\ Cali, Colombia. Contacto: sergey.gonzalez@correounivalle.edu.co \\ José Miguel Ramírez Scarpetta \\ Ingeniero electricista, máster en Ingeniería Eléctrica, máster en Automática-Pro- \\ dúctica, doctor en Automática, docente de la Universidad del Valle, Cali, Colom- \\ bia. Contacto: jose.ramirez@correounivalle.edu.co
}

\section{Edna Joydeth Avella Rodríguez}

Ingeniera electrónica, estudiante de Maestría de la Universidad del Valle, Cali, Colombia. Contacto: edna.avella@correounivalle.edu.co

Fecha de recepción: 22 de agosto del 2013

Clasificación del artículo: revisión

Fecha de aceptación: 15 de mayo del 2014

Financiamiento: Colciencias, Universidad del Valle

DOI: http://dx.doi.org/10.14483/udistrital.jour.tecnura.2015.1.a010

Palabras clave: equilibrio dinámico, equilibrio estático, locomoción, planos anatómicos.

Keywords: Dynamic equilibrium, locomotion, anatomical planes.

\section{RESUMEN}

Debido a la inestabilidad inherente de los robots bípedos, existen diferentes técnicas para controlarlos. El objetivo de este trabajo es presentar una revisión descriptiva de algunas técnicas de control para el equilibrio estático y dinámico $\mathrm{y}$ algoritmos para la generación de patrones de marcha desarrolladas en la robótica bípeda. El artículo también contiene una breve terminología de la locomoción bípeda. Las técnicas de control descritas se basan en la teoría de control a partir de modelos dinámicos de los robots bípedos.

\section{ABSTRACT}

There are different control techniques due to the inherent instability that bipedal robots have. The purpose of this paper is to present a descriptive review of some control techniques for static and dynamic balance; also, to check algorithms to generate gait patterns developed in bipedal robotics. The article also contains a brief glossary of bipedal locomotion. The control techniques described in this paper are based on the control theory from dynamical models of biped robots. 


\section{INTRODUCCIÓN}

La estrecha interacción entre los sistemas de manipulación robótica y los seres humanos ha generado que los robots se vuelvan comunes en ambientes no industriales como hogares, hospitales y áreas de servicio. Los robots bípedos se controlan de manera similar a los manipuladores industriales, con base en control de trayectorias. Para que el robot mantenga la estabilidad local, la marcha se debe sintetizar como una trayectoria continua a lo largo del espacio. Durante años se ha satisfecho esta restricción usando el paradigma del punto de momento cero (Santana Hernández, 2006). Se han propuesto otros criterios de estabilidad como el indicador de la rotación del pie, aunque la mayoría de los robots humanoides o bípedos utilizan el punto de momento cero. Una vez se garantice un criterio de estabilidad, el robot bípedo es capaz de caminar establemente. Esta revisión presenta de una forma básica algunos conceptos que abarcan la locomoción bípeda, luego se realiza una descripción de las técnicas de control de equilibrio aplicadas en robots bípedos, haciendo una clasificación entre el régimen estático y el dinámico.

\section{TERMINOLOGÍA EN LOCOMOCIÓN BÍPEDA}

Esta sección presenta algunos términos y acrónimos que serán utilizados en este artículo.

\section{Planos espaciales anatómicos}

Los tres ejes conforman los planos del espacio (Santana Hernández, 2006). El plano frontal o coronal se orienta de manera vertical, de forma tal que divide al cuerpo en anterior y posterior. El plano sagital se orienta verticalmente y perpendicular al plano frontal, dividiendo el cuerpo en dos zonas: derecha e izquierda, en tanto que el plano transversal o axial se orienta horizontalmente, a diferencia de los otros dos planos. De esta manera, el cuerpo se divide en zona inferior y zona superior.

\section{Centro de gravedad y centro de masa del cuerpo humano}

Desde la posición anatómica de pie, el centro de gravedad (CG) en el cuerpo humano se encuentra aproximadamente en la posición anterior de la segunda vértebra en el sacro. La ubicación precisa del vector de gravedad para una persona dependerá de sus dimensiones físicas, donde su magnitud es igual a la masa corporal del individuo. La ubicación del centro de masa o CM en el cuerpo humano es el punto donde hay mayor concentración de masa, coincide con el CG solo si el campo gravitatorio es uniforme (Zermeño Sobrino, 2006).

\section{Centros de gravedad de los segmentos}

Cada segmento del cuerpo humano posee su propio centro de gravedad. Esto quiere decir que sobre estos existe una fuerza de gravedad. En el caso de que dos segmentos adyacentes se combinen para ejercer una fuerza o movimiento, se consideran como un solo segmento sólido. Entonces el nuevo segmento tendrá un nuevo centro de gravedad que estará ubicado y alineado en el medio de los centros de gravedad originales (Zermeño Sobrino, 2006).

\section{Polígono de soporte}

El polígono de soporte (apoyo) o área de soporte (AS) es una figura geométrica plana formada en el suelo por uno o dos pies (Santana Hernández, 2006). En caso de soporte sobre un único pie, es este mismo. En caso de soporte con los dos pies, se abarca la región de los pies y la parte del área entre ambos. 


\section{Proyección del centro de masa y centro de presión}

La proyección del centro de masa es el punto obtenido al proyectar verticalmente el CM sobre el AS (frecuentemente el plano horizontal), mientras que la proyección del centro de presión $(\mathrm{CP})$ es el punto obtenido al proyectar el CM en la dirección de la resultante de fuerzas del sistema sobre el AS (Santana Hernández, 2006).

\section{Punto de momento cero}

El punto de momento cero (PMC) es el punto sobre el AS del humanoide donde se anula el momento resultante de las fuerzas externas, la gravedad y las fuerzas de inercia (Santana Hernández, 2006). Se define también como el punto en el suelo tal que el momento neto de las fuerzas externas no tiene componentes sobre los ejes horizontales. Cuando existe dentro del AS, el contacto entre el suelo y el pie es estable. Cuanto más cercano esté al centro de la superficie de soporte, más robustez se conseguirá. Cuando está fuera del AS, el robot se inclina rotando sobre alguno de los bordes de dicho polígono. El criterio de que el PMC exista dentro del AS es condición necesaria y suficiente para garantizar la estabilidad dinámica del robot (Fernández Iglesias, 2009)

\section{Centro de presión}

El centro de presión (CP) es el punto en la superficie de contacto donde se considera que actúa la fuerza de reacción neta del piso (Santana Hernández, 2006).

\section{Índice de rotación del pie}

El índice de rotación del pie (IRP) es el punto en la superficie en el cual la reacción del suelo tiene que actuar para que el pie permanezca estacionario (Santana Hernández, 2006).

\section{Margen de estabilidad estática y margen de estabilidad dinámica}

Para un determinado momento, el margen de estabilidad estática es la distancia más corta entre la proyección del centro de masa y el polígono de soporte, en tanto que el margen de estabilidad dinámica es la distancia más corta entre la proyección del centro de presiones y el polígono de soporte. Es la mínima distancia entre el PMC y los bordes del polígono de soporte, en donde SMx es el margen de estabilidad en el eje $\mathrm{x}, \mathrm{y} \mathrm{SMz}$ es el margen de estabilidad en el eje z (Santana Hernández, 2006).

\section{Equilibrio estático y equilibrio dinámico}

Existen dos modelos básicos de equilibrio (balanceo) en la locomoción bípeda: el "equilibrio estático" y el "equilibrio dinámico". El equilibrio estático lleva a movimientos lentos y menos naturales, mientras que para ganar eficiencia y velocidad se tiene que conseguir un equilibrio dinámico. Los dos tipos de equilibrio se basan en la implementación de leyes de control que utilizan el concepto de AS (Pardos Gotor, 2005).

\section{Ciclo de marcha}

El ciclo de la marcha inicia cuando el pie hace contacto con el suelo y finaliza cuando este mismo pie hace contacto nuevamente con el suelo. El ciclo de marcha o caminata es un tipo de movimiento periódico y está descrito por dos fases principales que se alternan con cada pierna: la fase de apoyo y la fase de balanceo. Una pierna se encuentra en fase de apoyo cuando está en contacto con el suelo, y se encuentra en fase de balanceo cuando no tiene contacto con el suelo (Guzmán Valdivia, 2010). 


\section{TÉCNICAS DE CONTROL APLICADAS A ROBOTS BÍPEDOS}

En la teoría de la locomoción bípeda para los robots humanoides, la marcha se puede aplicar en cualquier tipo de terreno, por eso se estudia solamente el uso de robots humanoides en ambientes humanos así como el diseño de algoritmos de control para el balanceo y la marcha aplicados a este tipo de robots. La mayoría de los investigadores trabajan con robots humanoides de bajo costo, ya que estos son más accesibles y también ofrecen la oportunidad de ser estudiados para implementar nuevos algoritmos de equilibrio y marcha.

Esta revisión manifiesta que los diseños de los robots humanoides difieren en restricciones, cantidad, tipo, tamaño, tiempo de respuesta de los actuadores, sensores e incluso en su estructura mecánica. Todo esto da una idea de la posible dinámica de marcha en el robot, así como de una variedad de técnicas de control para equilibrio y métodos de planificación o generación de trayectorias suaves para lograr un balance dinámico. Hoy en proyectos de investigación se usa la medición de la inclinación de postura y la desviación del centro de masa como realimentación a las estrategias de control. Estas estrategias utilizan diferentes tipos de sensores y algoritmos para procesar los datos recibidos, a fin de estimar la inclinación y la posición del centro de masa de forma precisa y aplicar las acciones de control indicadas.

La revisión establece una clasificación entre los trabajos que enfatizan en el diseño de algoritmos de control para equilibrio estático-dinámico y los métodos de generación de patrones de marcha aplicados a robots bípedos. Los trabajos se realizaron en universidades y laboratorios de investigación, los resultados han sido publicados en artículos de diferentes revistas y eventos.

\section{Algoritmos de control para equilibrio estático y dinámico aplicados en robots bípedos}

Esta sección expone técnicas de control aplicadas a robots humanoides para lograr el equilibrio estático y dinámico. Estas técnicas se clasifican dependiendo del tipo de equilibrio deseado.

Técnicas de control para equilibrio estático. La revisión realizada presenta algoritmos para el control de equilibrio estático. Aun así, se describen los trabajos hallados que desarrollan técnicas de control para el régimen estático.

Los robots bípedos en su mayoría poseen actuadores en los tobillos, haciendo más fácil el diseño del control en la caminata. El trabajo de Abdallah y Goswami (2005) presenta una estrategia de control de dos fases para el mantenimiento de equilibrio robusto bajo una fuerza de perturbación. La primera fase es llamada la fase de reflejo, se diseña para resistir el efecto inmediato de una fuerza, donde la ley de control utilizada es el momento angular; se caracteriza por resistir a la perturbación. La segunda fase es la recuperación, donde el sistema es dirigido a una posición "home" donde es estáticamente estable. La ley de control es de recuperación, emplea un esquema general que maximiza la energía potencial con diferentes superficies de terreno.

El objetivo de un robot bípedo es recuperar y mantener el balance mediante la detección de disturbios externos ejecutando los reflejos. Como lo explican Corpuz, Lafoteza, Broas y Ramos (2009), la instrumentación utilizada es un acelerómetro y un giróscopo que permiten estimar la postura del sistema, a su vez se utiliza un filtro Kalman como algoritmo de fusión de las señales del acelerómetro y giróscopo para tener una medida de la orientación. El algoritmo de equilibrio utilizado es un péndulo invertido de doble soporte. Los límites del YICAL se establecen por la velocidad y máxima inclinación. El algoritmo 
usa supuestos como lo son: mantenerse en posición de doble apoyo, la posición de la cadera con respecto a los muslos es fija.

Sheng-jun, Hai-tao, TaoyHong-xu (2009) proponen el grado de pérdida de equilibrio como un criterio de estabilidad para robots bípedos subactuados. Considerando que el robot bípedo no tiene pies, se analiza que el contacto con tierra se realiza puntual, modelándolo como un péndulo invertido con longitud variable y momento de inercia. Para el cálculo del grado de pérdida de equilibrio se utiliza el modelo lineal, en el que se tiene un punto de equilibrio inestable y la tarea principal del control es mantener el estado del robot cerca de la vecindad del punto de equilibrio y evitar la caída.

Ono, Sato y Ohnishi (2011) proponen las estrategias de recuperación de equilibrio y el cambio de las estrategias basadas en el valor de la aceleración del CG. Los robots humanoides son sometidos a una fuerza externa repentina mientras están de pie. De acuerdo con la fuerza externa aplicada, se determina qué estrategia se usa, como se observa en la figura 1. Este documento asume que el valor de la fuerza externa es equivalente al valor de la aceleración del CG, el cual determina si la articulación de la rodilla se usa para la recuperación del equilibrio

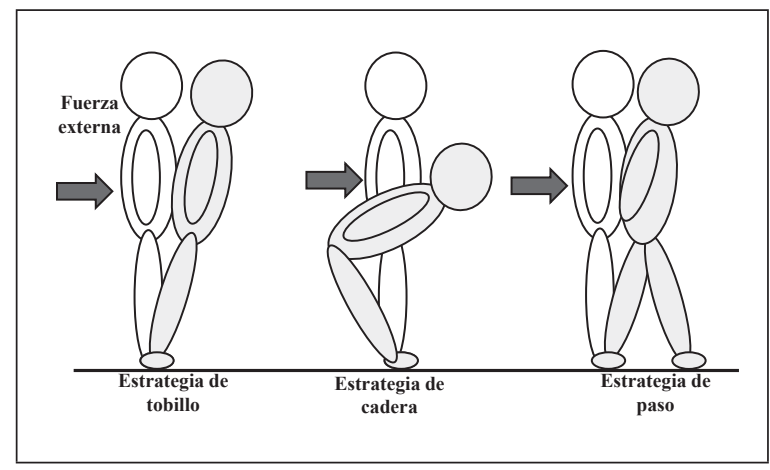

Figura 1. Tres estrategias básicas de balanceo Fuente: Ono et ál. (2011).
Kim et ál. (2012) presentan el control de equilibrio para un robot bípedo con control de par, esta técnica se aplica en el robot Roboray, el cual tiene seis grados de libertad (GDL) y sensores de par en todas las articulaciones de las piernas. Asimismo, tiene un módulo de empalme de cable impulsado como una unidad de articulación de cabeceo. Con el uso de estas características se propone un algoritmo de control para balanceo. Este algoritmo es la combinación de compensación de gravedad, control de la gravedad virtual y control de la amortiguación. También se introduce una técnica de compensación de fricción, con el fin de eliminar la no linealidad de amortiguación y mejorar el rendimiento de seguimiento de par. El método propuesto se aplica a un sistema de péndulo invertido simple y al robot Roboray.

Roa y Ott (2013), por su parte, presentan una visión general de un enfoque para el equilibrio y control de postura, regulando al mismo tiempo la posición del CM y la orientación del tronco de un robot bípedo. Al realizar una perturbación externa el robot se desvía de la postura deseada, el controlador calcula una fuerza-par necesaria para recuperar la posición y la orientación deseadas, de acuerdo con la ley de control. Esta fuerza-par se distribuye a los puntos de contacto de soporte en los pies. Las fuerzas en estos puntos se calculan a través de un problema de optimización con restricciones, lo que minimiza las fuerzas de contacto, mientras que al incluir las restricciones y límites de par existe una fricción en cada junta.

Técnicas de control para equilibrio dinámico. El equilibrio dinámico es el objetivo general en la robótica bípeda, ya que evita la caída inminente del robot bípedo cuando experimenta grandes perturbaciones; por consiguiente, le permite interactuar en terrenos agrestes. Para lograrlo se necesitan diversos sensores que den información útil del estado del sistema bípedo, como es el centro de presión en los pies, torques, velocidades y 
posiciones angulares de las articulaciones, aceleraciones del centro de masa, etc.

A continuación se explican desarrollos de estrategias de control aplicados a robots bípedos para obtener el equilibrio dinámico, estas técnicas se aplican a robots bípedos construidos; otros solamente se proponen a nivel de simulación.

Yeoun-Jae, Joon-Yong y Ju-Jang (2012) proponen una estrategia de control de balance para un robot bípedo mientras camina, aplicando una fuerza externa. Se parte de la suposición de que durante la marcha se aplican una fuerza lateral y una longitudinal en el centro de la pelvis del robot bípedo, para este análisis se tiene en cuenta la eficiencia de la energía.

Liu, Li, Guo y Cai (2012), a su vez, proponen un sistema de control donde se tratan la postura corporal, la velocidad y el giro de la pierna de balanceo como tres problemas de control independientes. La primera parte del control mantiene el cuerpo en posición vertical, teniendo en cuenta el par de la cadera de la pierna de apoyo. La segunda parte del control estabiliza la velocidad de avance mediante la regulación de la longitud de la pierna de apoyo, en tanto que la tercera parte es el control del ángulo de la pierna de balanceo.

Li, Su y Liu (2012) desarrollan un nuevo modelo de trayectoria con parámetros ajustables que modula la trayectoria con PMC en los planos sagital y lateral y hace que la trayectoria del PMC sea más flexible. La dinámica de control incluye control de equilibrio dinámico, un filtro de Kalman y un controlador de movimiento diseñado para mantener el equilibrio del cuerpo y permitir que el bípedo camine siguiendo la referencia del PMC deseada.

Li y Ge (2012) muestran una estructura de control adaptativo robusto que incluye el equilibrio y un control postural para regular la posición del CM y la orientación del tronco de los robots bípedos de una manera compatible. En primer lugar, el robot bípedo se desacopla de la dinámica del CM y el tronco. El control calcula la fuerza de reacción del suelo para estabilizar la postura con la dinámica desconocida del CM y luego transforma estas fuerzas en pares de conjuntos de todo el cuerpo, incluso si existen las perturbaciones externas.

Guihard y Gorce (2002) presentan una forma de resolver el control dinámico bajo perturbaciones desconocidas que tiene en cuenta la noción de movimiento postural. Para ello se propone un modelo mecánico multicadena de un cuerpo humano y una arquitectura de control general. Además, se utiliza una estrategia postural de tobillo y cadera donde la noción de movimiento asegura el equilibrio dinámico bípedo.

En el trabajo de Lim, Setiawan y Takanishi (2001) se propone un control de impedancia y un control de equilibrio para robots bípedos, a fin de que caminen de forma estable y en condiciones como las de un ser humano. El control de equilibrio se basa en el movimiento del tronco y la cintura, lo cual esto se aplica para mantener el equilibrio y la postura, mientras que el movimiento de las extremidades inferiores se controla de acuerdo con el terreno. El control de impedancia se aplica para reducir la fuer$\mathrm{za} /$ torque al realizar el contacto entre el pie y el suelo. Los parámetros de la impedancia se cambian en tiempo real, dependiendo de la etapa de la marcha.

Pratt, Chew, Torres, Dilworth y Pratt (2001) utilizan un modelo de control virtual. Es un lenguaje de control de movimiento que utiliza simulaciones, son componentes mecánicos para crear fuerzas que se aplican a través de pares de conjuntos reales, creando la ilusión de que los componentes virtuales están conectados al robot.

Rehbinder y Hu (2001) muestran que una de las técnicas empleadas en el control de robots móviles es la estimación del estado, donde los estados son típicamente posición, velocidad y orientación. 
Los dispositivos para realizar esta estimación son un giróscopo y un acelerómetro de tres ejes, a fin de dar estimaciones estables de la postura del robot. Se usa una descripción global de la rotación del cuerpo-rígido para obtener un problema lineal y poder usar un filtro Kalman lineal modificado. De esta forma se logra que no haya restricción en el tipo de movimientos que puede realizar el robot.

La Universidad de Grenoble en Francia construyó el robot bípedo Rabbit (Grizzle, 2002), el cual carece de pies, las piernas terminan como zancos de manera que oscila sobre un punto cuando avanza. El concepto de estabilidad es representado con fórmulas matemáticas que comprenden la dinámica de la marcha y el equilibrio, produciendo automáticamente un algoritmo de control que induce a un movimiento deseado. Se puede variar la velocidad de la marcha y se utiliza conmutación de leyes de control y una acción de control integral.

La investigación de Chevallereau et ál. (2003) muestra el diseño de control mediante restricciones virtuales para la fase de balanceo del robot Rabbit. El robot tiene cinco segmentos, cuatro actuadores, es planar y realiza la marcha bípeda. El diseño del control considera al robot en la fase de único soporte y las restricciones se realizan de acuerdo con un elemento común que es la regulación de las cuatro salidas independientes (actuadores); la elección de los actuadores se realiza para regular el ángulo del torso, la altura de las caderas y la posición final de la pierna en balanceo.

La mayoría de las estrategias de control de un robot están construidas alrededor del seguimiento de trayectorias, que se generan ya sea fuera de línea, durante la fase de planificación de trayectoria, o en línea, por medio de un planificador de movimiento de alto nivel. Chevallereau et ál. (2003) proponen que para controlar adecuadamente el robot Rabbit se necesita una teoría de control para una clase de sistemas con una dinámica tanto continua como discreta y menos actuadores que grados de libertad; además, se desean órbitas asintóticamente estables, en lugar de puntos de equilibrio, y tener múltiples objetivos como caminar, correr y equilibrar. En la figura 2 se observa la implementación del sistema de control sobre el robot bípedo Rabbit basado en restricciones virtuales y dinámica híbrida cero para la marcha. Otro análisis de Chevallereau, Grizzle y Shih (2009) presenta controladores que permiten una caminata asintóticamente estable, periódica y rápida para un robot bípedo. La primera estrategia consiste en imponer una condición de estabilidad durante la búsqueda de la caminata por optimización periódica. La segunda estrategia utilizada es un controlador basado en eventos para modificar los valores propios del mapa de Poincaré. En el tercer enfoque se analiza el efecto de selección de la salida en la dinámica de cero y se propone una selección pertinente de los productos, lo que lleva a la estabilización sin el uso de un controlador basado en eventos suplementarios.

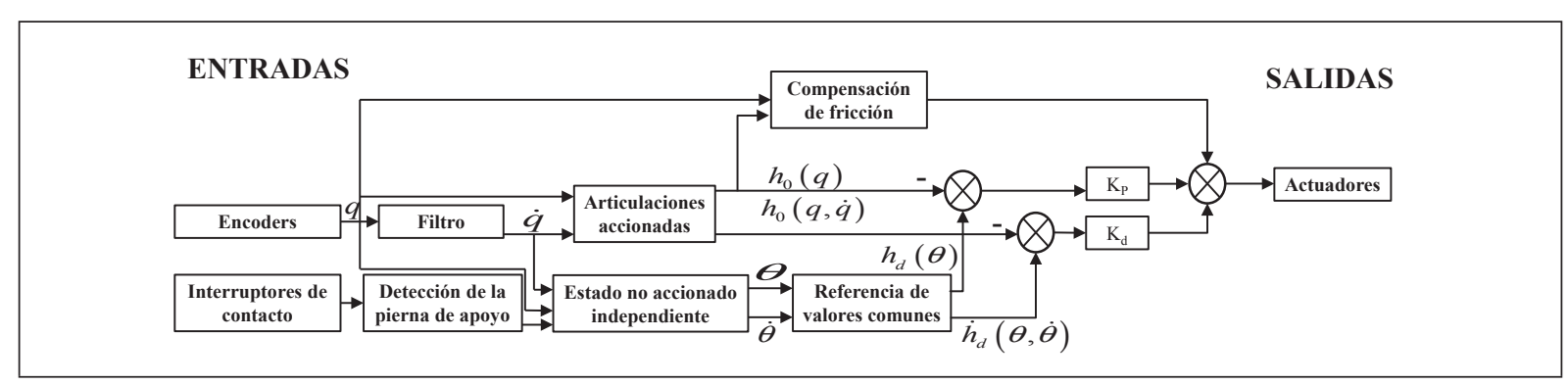

Figura 2. Diagrama de bloques del sistema de control -Rabbit

Fuente: Chevallereau et ál. (2003). 
Canudas-de-Wit (2004) revisa una clase de métodos de control para el control de marcha y el problema de equilibrio. Los métodos en estudio se basan en la noción de restricciones virtuales que fuerzan la realimentación, como se observa en la figura 3. Las restricciones son las relaciones entre los eslabones del mecanismo que están impuestas dinámicamente a través del control de realimentación. Su función es coordinar la evolución de los distintos eslabones mediante un paso; por lo tanto, se reducen grados de libertad con el objetivo de lograr un mecanismo de lazo cerrado que naturalmente dé origen a un movimiento periódico deseado. Para el problema de equilibrio tratan al robot en la fase de único soporte como un péndulo invertido multienlace.

La investigación de Santana Hernández (2006) enseña un robot bípedo, con sensores como: sensores de fuerza, giroscopios, acelerómetros, etc. Describe cómo se realiza la marcha para un robot bípedo que se encuentra dinámicamente estable durante su caminata, los parámetros como el PMC o el $\mathrm{CM}$ se encuentran dentro del rango de estabilidad. En el momento en que estos parámetros sa- len del rango de estabilidad se realizan las acciones correctivas para que el robot evite caer.

La investigación de Manrique (García, 2007) muestra técnicas de control en línea, las cuales se orientan a garantizar un movimiento dinámicamente equilibrado, por lo que es necesario el estado del entorno y la posición del PMC. Para asegurar un equilibrio completo, estas estrategias deben ser utilizadas en forma cooperativa; primero el control de secuencia de movimientos reajusta la posición del robot generando un paso, cuya longitud y dirección modifica o aumenta el área de soporte del humanoide. Segundo, el control de par, que consiste en la generación de un torque alrededor del CM para hacer mover el PMC hacia atrás, se utiliza cuando el control del PMC no es efectivo.

Kwon et ál. (2007) generan un algoritmo de control de marcha que consiste en la generación de patrones de marcha, control de impedancia para el apoyo y marcha sobre una superficie irregular y el control del PMC en tiempo real para el control de reflejo (figura 4). El algoritmo no se ha demostrado en experimentos.

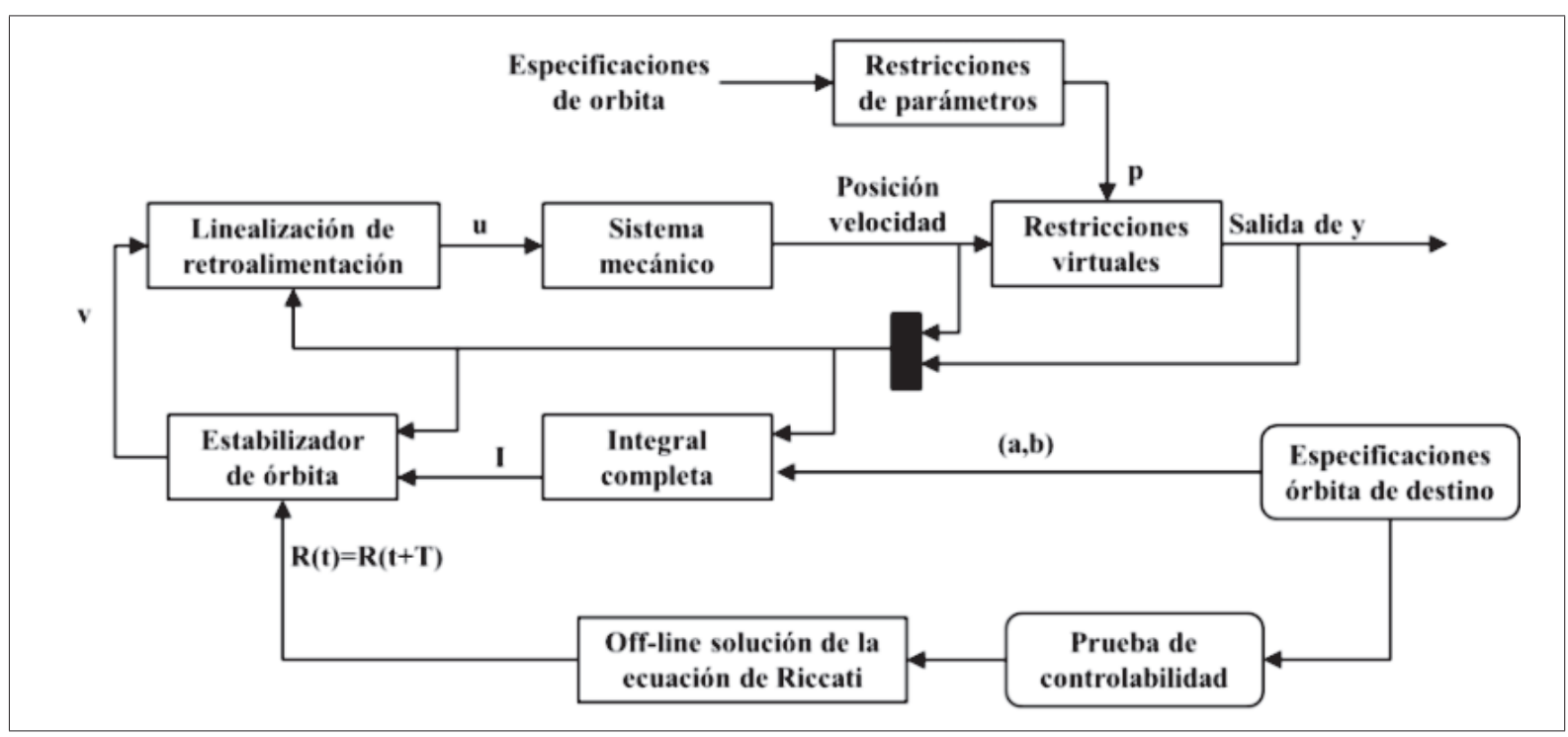

Figura 3. Diagrama de bloques del estabilizador orbital

Fuente: Canudas-de-Wit (2004). 
Se han desarrollado sistemas de control híbrido y controladores de compensación para sistemas con múltiples entradas y múltiples salidas (MIMO) no lineales con incertidumbres. Lin, Chen y Chen (2007) afirman que un sistema de control híbrido se basa en la técnica de modos deslizantes y utilizan un controlador de articulación por modelo de cerebelo recurrente (RCMAC) como un observador de incertidumbre. En la figura 5 se observa que el controlador principal contiene un observador de incertidumbre RCMAC, siendo este el controlador principal, y un controlador de compensación para la aproximación de error del sistema incierto.

Shih, Grizzle y Chevallereau (2007) presentan un controlador por retroalimentación que logra una marcha asintóticamente estable, periódica y rápida para un robot bípedo en 3D, que consta de tres enlaces y pies puntuales pasivos. Cada enlace es modelado por una masa puntual en su centro y no hay movimiento de viraje sobre el final de la pierna de apoyo. Asumen un contacto puntual no actuado en el final de la pierna y buscan un controlador por realimentación invariante en el tiempo que crea un movimiento exponencialmente estable y periódico.

Uno de los desarrollos es el robot de Honda Asi$\mathrm{mo}^{1}$ (Honda Motor, 2007), que se desarrolla para funcionar en la sociedad y en un entorno de vida humana; se realiza un prototipo de tamaño casi humano. Cuando Asimo pierde el equilibrio y trata de caer, los tres siguientes sistemas de control, como se muestran en la figura 6 , funcionan para evitar la caída y permitir una caminata continua. Primero un control de reacción del piso que absorbe las irregularidades del suelo y controla la ubicación de las plantas de los pies cuando la caída es inminente; segundo, el control para referencia de PMC, y tercero, el control de posicionamiento de la planta del pie.

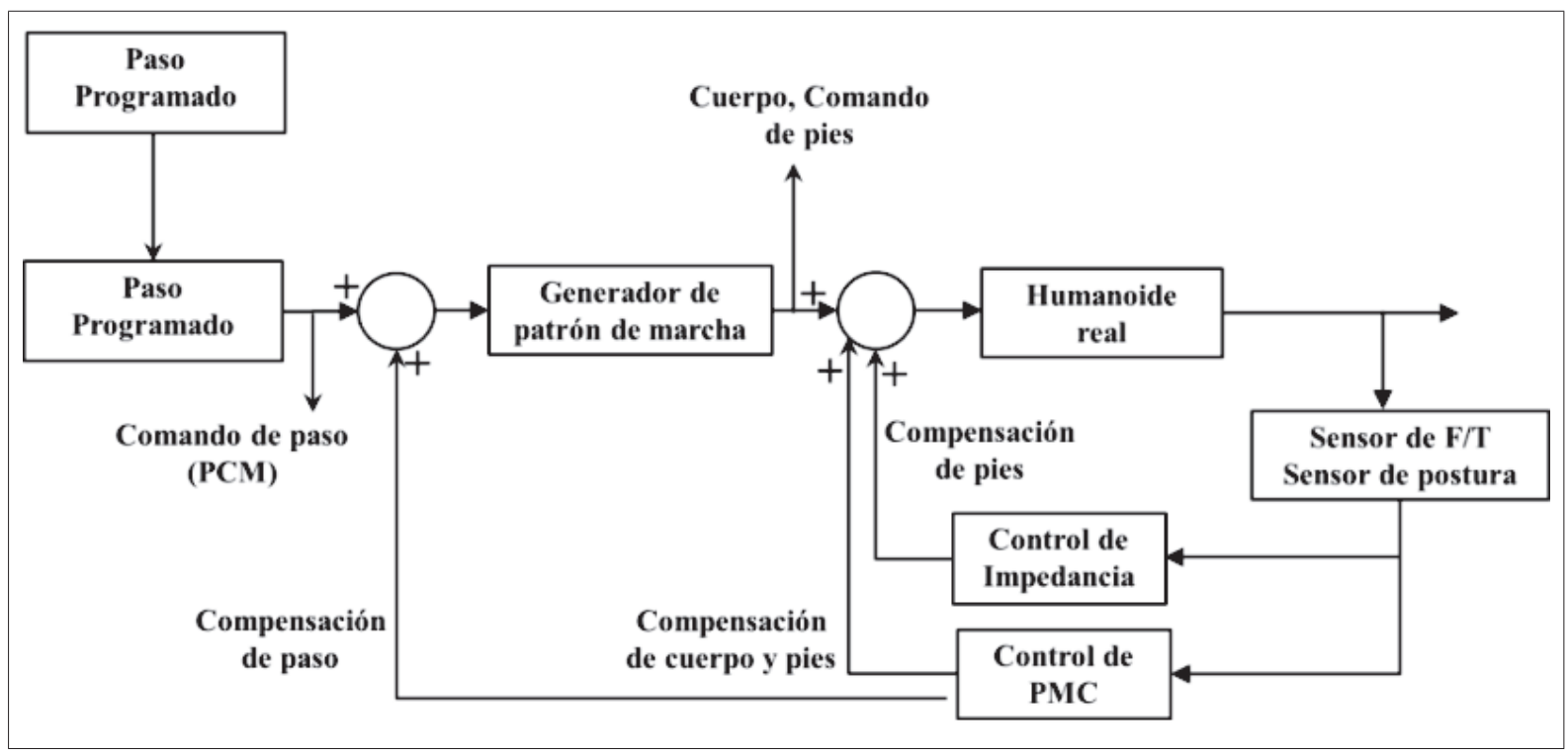

Figura 4. Diagrama del algoritmo de control de marcha

Fuente: Kwon et ál. (2007).

1 Advanced Step in Innovative Mobility. 


\section{revisión}

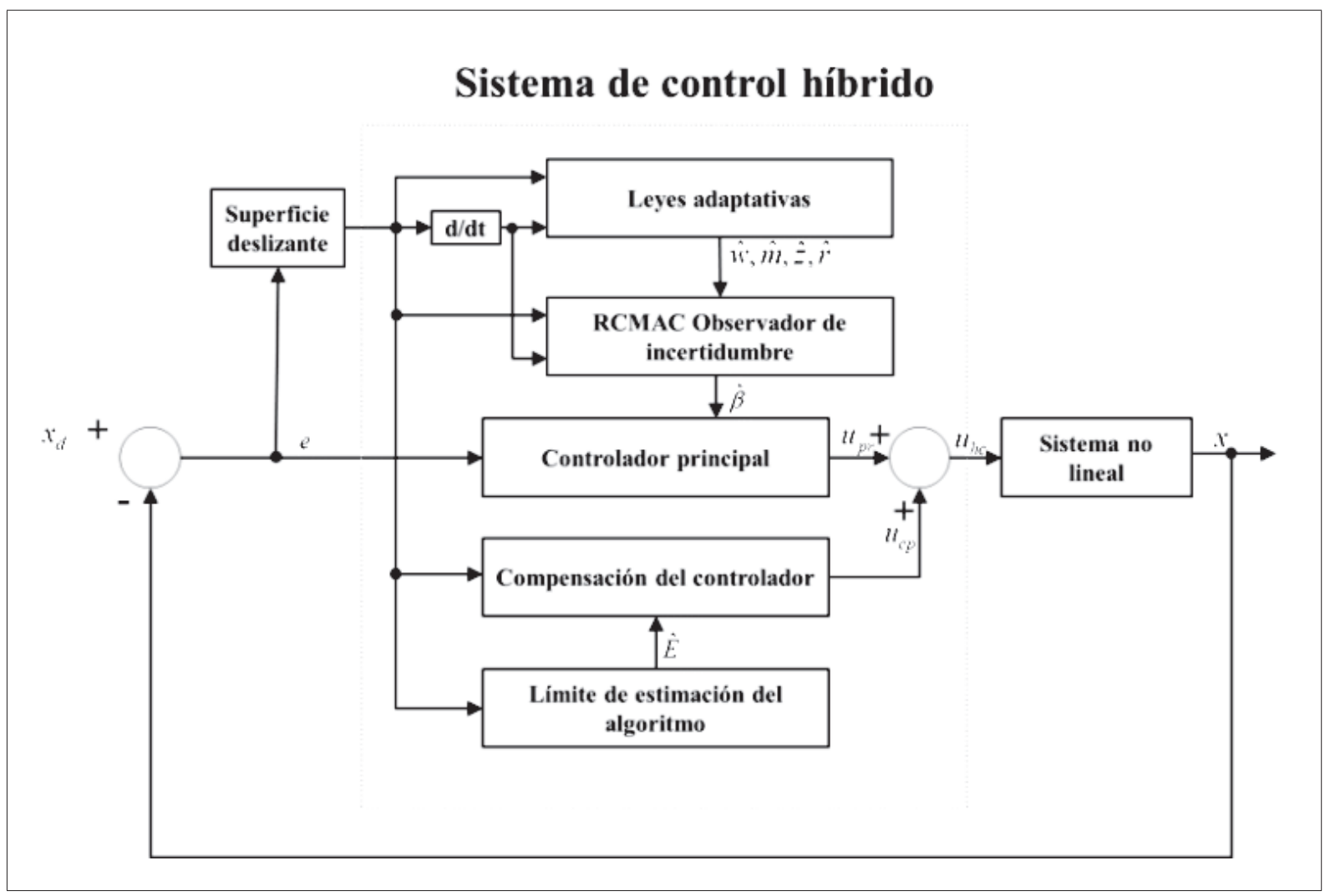

Figura 5. Sistema de control hibrido con RCMAC

Fuente: Lin et ál. (2007).

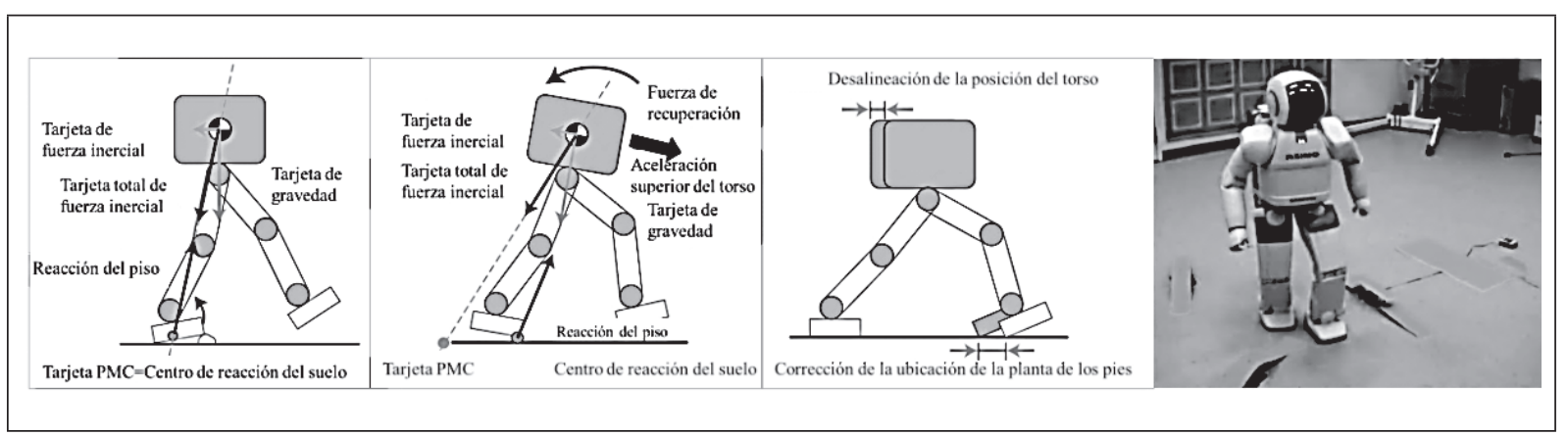

Figura 6. Sistemas de control para el robot Asimo

Fuente: Honda Motor (2007).

La compañía Anybots (2007) presenta a sus dos robots, llamados Dexter y Monty, estos aprenden basados en ensayo y error. Monty es un robot humanoide que atrapa una pelota y la lanza, acción esta que demuestra una estabilidad de la base con ruedas equilibradas. Este robot tiene el mismo sistema del Segway donde se controla la proyección del CM para que esté dentro de la región de 
soporte que forman las dos ruedas. Dexter, el robot caminante, realiza un equilibrio dinámico para caminar, la marcha mejora con la práctica. Cuando está cayendo, el robot conoce la dirección y apoya el pie en el lugar exacto para balancear en la dirección que se desea ir, también la acción de caer se hace porque el CP no se centra sobre el pie (o pies) que está en el suelo.

La Universidad de Michigan desarrolla al robot bípedo Mabel (Grizzle, 2008), un robot que tiene un tronco pesado, piernas ligeras y flexibles, con una serie de muelles (resortes) que actúan como tendones. Se conoce como el robot bípedo más rápido del mundo, capaz de alcanzar casi 11 kilómetros por hora. Está en el aire el $40 \%$ de cada paso, su cuerpo cuenta con un gran torso y delgadas piernas que tienen rodillas, las cuales le permiten correr, y se sostiene por medio de una barra mientras corre en círculos por el lugar (Matt, Shane, Allison y Philip, 2010).

En la investigación de Aclan y Ramos (2009) se implementa un control PID multivariable, aplicado al robot YICAL II, que tiene seis grados de libertad y es de accionamiento directo con motores de corriente continua.

Para la marcha bípeda dinámica se propone un algoritmo realizado por Xin y Xiao (2009), este (figura 7) incluye el cálculo del movimiento de la parte superior del cuerpo y la trayectoria del CG del robot. Los disturbios del ambiente son eliminados por el movimiento compensatorio de la parte superior del cuerpo; posteriormente, el error del CG se calcula basado en el error entre el PMC deseado y el PMC real, así como la relación entre el PMC y el CG para obtener la trayectoria del CG. Entonces, el movimiento del robot converge a su movimiento de referencia generando marcha bípeda estable.

Matsumoto y Kawamura (2010) proponen un sistema de control robusto de dirección para am- bientes con perturbaciones, usando la medida de un giróscopo, ya que puede ser necesario un giro del robot en el espacio de trabajo. En el modelo de control se corrige el ángulo de postura del robot por una acción integral, así como el ángulo de dirección antes de dar el paso. Los autores proponen el control robusto de dirección para mejorar la respuesta debido a disturbios (ver figura 8). Esta técnica tiene un controlador proporcionalderivativo PD en realimentación y en adelanto para la fase de soporte.

Ferreira, Crisóstomo y Coimbra (2010) describen el control de un robot bípedo autónomo, el cual combina el uso de las articulaciones del torso y del tobillo para el equilibrio sagital, como se observa en la figura 9. Este controlador combina las articulaciones del tobillo y el torso a fin de corregir el PMC. Para esto utiliza una técnica de inteligencia artificial, regresión de vectores de soporte, con el objeto de controlar el equilibrio del robot. Estos resultados fueron probados en superficies planas y en superficies inclinadas.

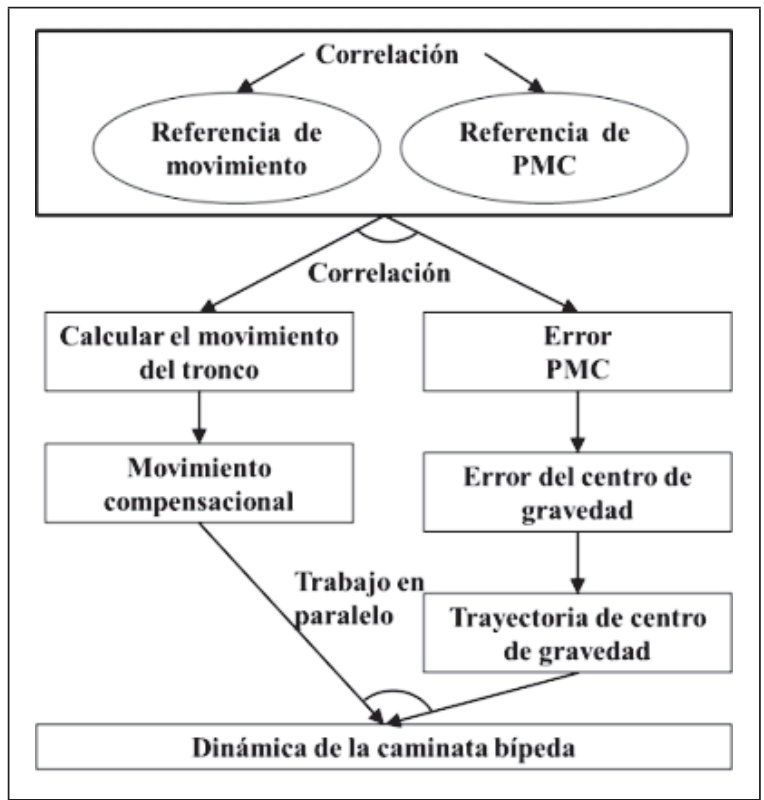

Figura 7. Algoritmo compuesto

Fuente: Xin y Xiao (2009). 


\section{revisión}

Parsa y Farrokhi (2010) analizan el uso de un observador no lineal de perturbaciones, para hacer más robusto un control predictivo basado en un modelo no lineal en robots bípedos. Los autores consideran la robustez del controlador para in- certidumbres en las dinámicas o variaciones de los parámetros del modelo del bípedo. Para robustecer el controlador se utiliza un observador no lineal de perturbación, como se observa en la figura 10 .

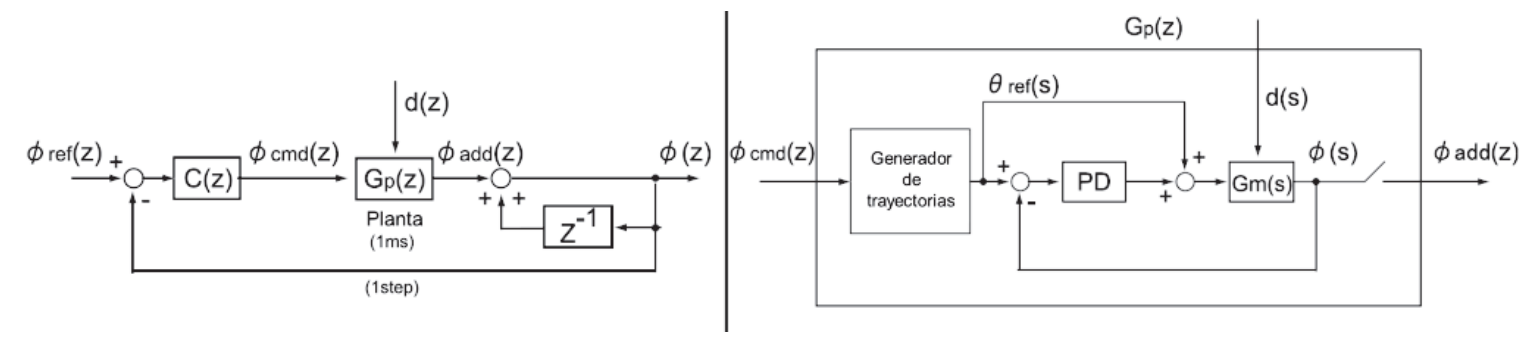

Figura 8. Modelo de control y controlador propuesto

Fuente: Matsumoto y Kawamura (2010).

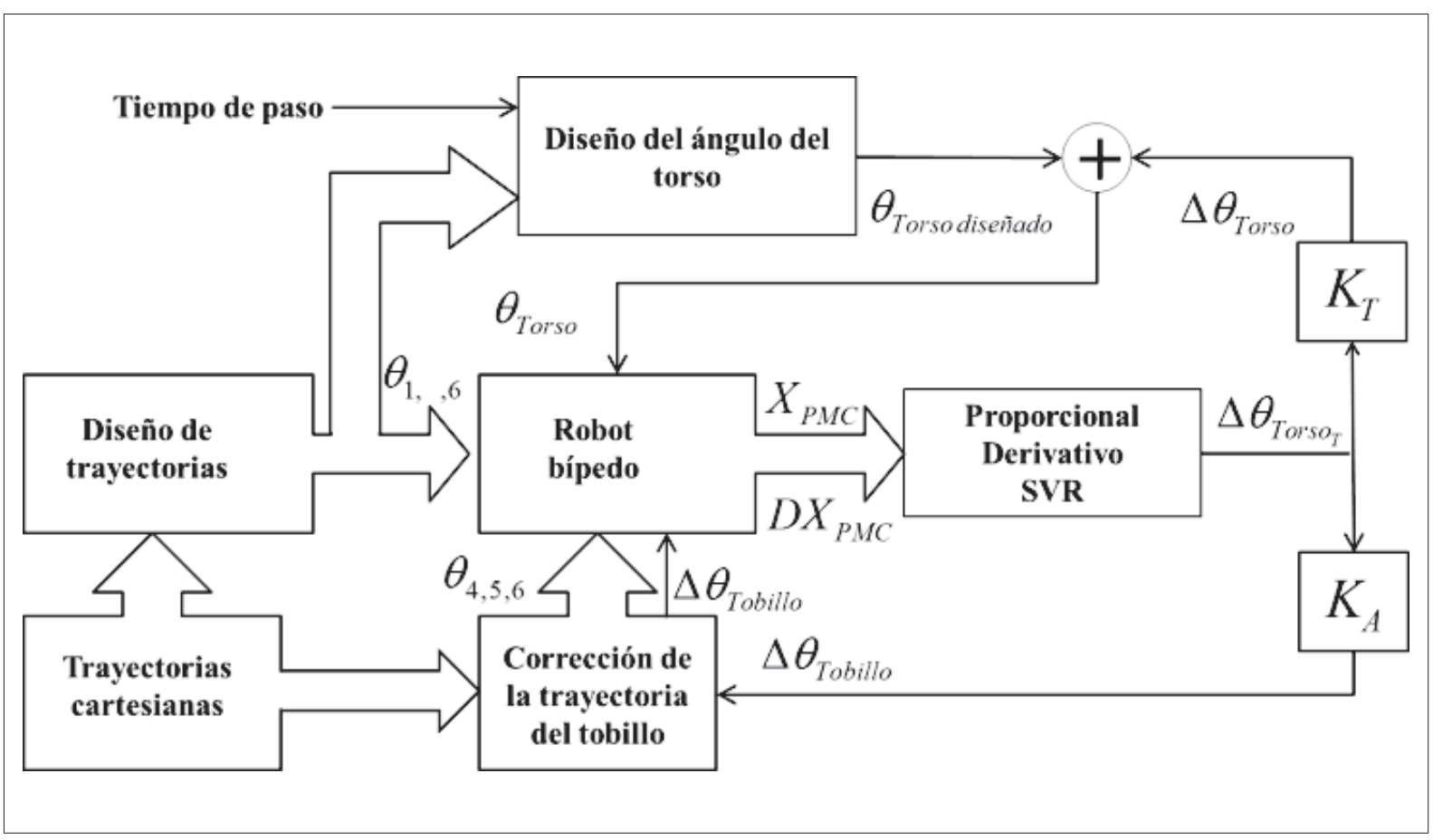

Figura 9. Control para el ángulo del torso y tobillo en el robot

Fuente: Ferreira et ál. (2010). 


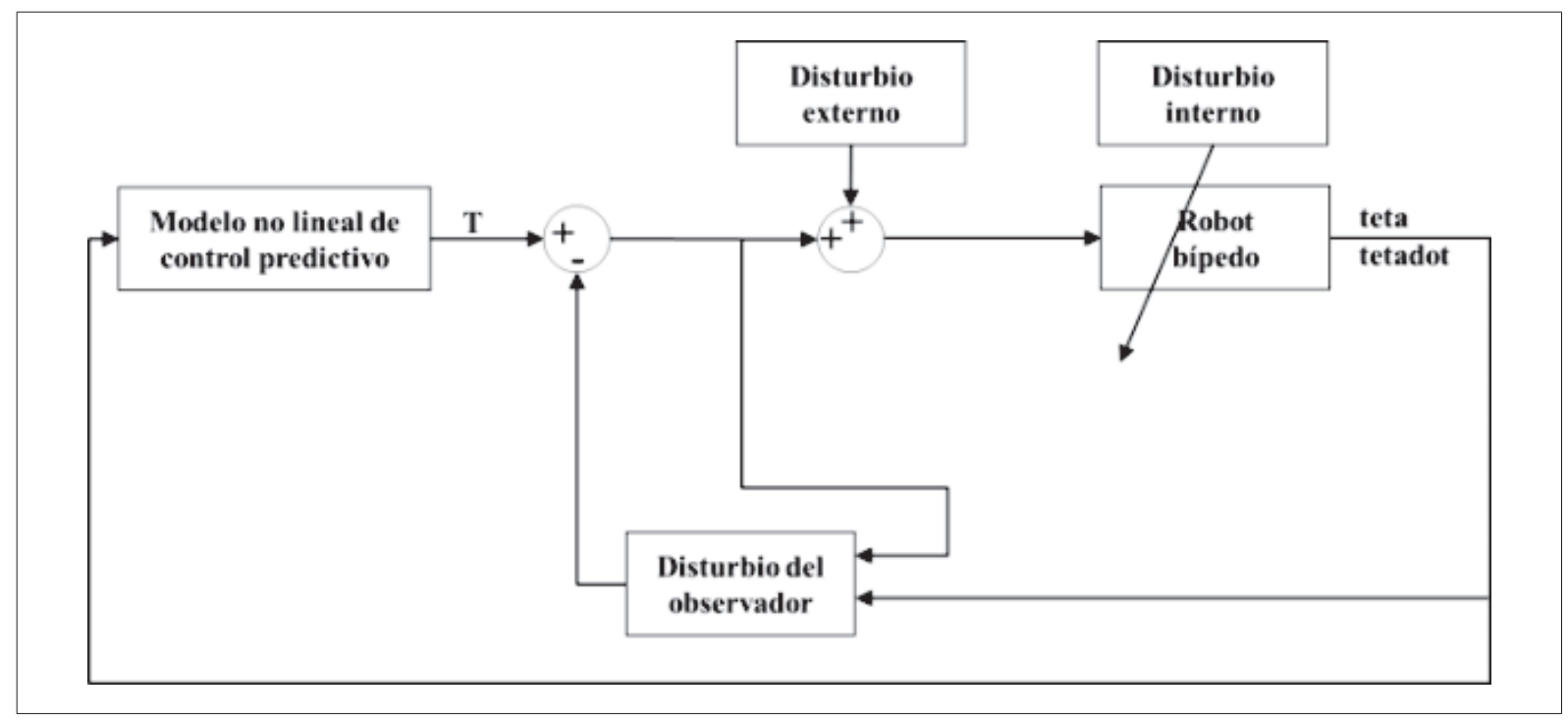

Figura 10. Estructura del observador no lineal de disturbios

Fuente: Parsa y Farrokhi (2010).

Los métodos propuestos por Aghabalaie, Hosseinzadeh, Talebi y Shafiee (2010) se basan en el control de todas las articulaciones, en lugar de controlar solamente la cadera y el tronco. La metodología de diseño para el controlador robusto utiliza el método de Lyapunov. Las restricciones de fuerzas debido a resbalones y levantamiento de los pies se consideran como perturbaciones adicionales que actúan en la dinámica del robot. En la ecuación 1 se observa la ley de control, esta se encuentra en función de los estados y la dinámica del robot.

$U=(I-C)^{-1}\left[\begin{array}{l}-(I+P-M(q)) x_{1}-(M(q)-Q) \\ x_{2}+D-k x_{2}^{T}+2 P_{2}^{2} x_{1}\end{array}\right]_{(1)}$

En la investigación realizada por Li et ál. (2010) se desarrolla una estructura de control estabilizante que consiste en un sistema sensorial piefuerza y controladores para marcha con respuesta dinámica rápida. Este sistema consta de tres controladores, como se evidencia en la figura 11: el controlador adaptativo, que controla el tobillo del pie que aterriza para lograr un aterrizaje suave y un contacto estable con el suelo; el controlador de equilibrio, a fin de mantener el PMC actual dentro de la región estable deseada. Este se usa para ajustar el tobillo de la pierna de soporte y asegurar una distancia entre el PMC actual y el límite de la región estable; por último, el controlador de retorno evalúa si el PMC actual está dentro de la región estable deseada o si la pierna se encuentra en la fase de balanceo. En este caso el patrón de la marcha debe retornar al patrón dinámico original. De lo contrario, los ajustes de la articulación del tobillo serán los errores acumulados por el patrón de la marcha dinámica original.

La estructura de control desarrollada en Rokbani, Benbousaada, Ammar y Alimi (2010) tiene un controlador de torso superior y se encuentra compuesta por dos submódulos: el módulo de control de las articulaciones y el controlador de torso superior. Este último ayuda a la estabilidad de la marcha corrigiendo la posición del CM del robot. $\mathrm{La}$ arquitectura propuesta integra principalmente tres módulos, como se observa en la figura 12: generador de marcha, módulo de adaptación marcha y el controlador de torso superior para controlar la estabilidad de marcha. 


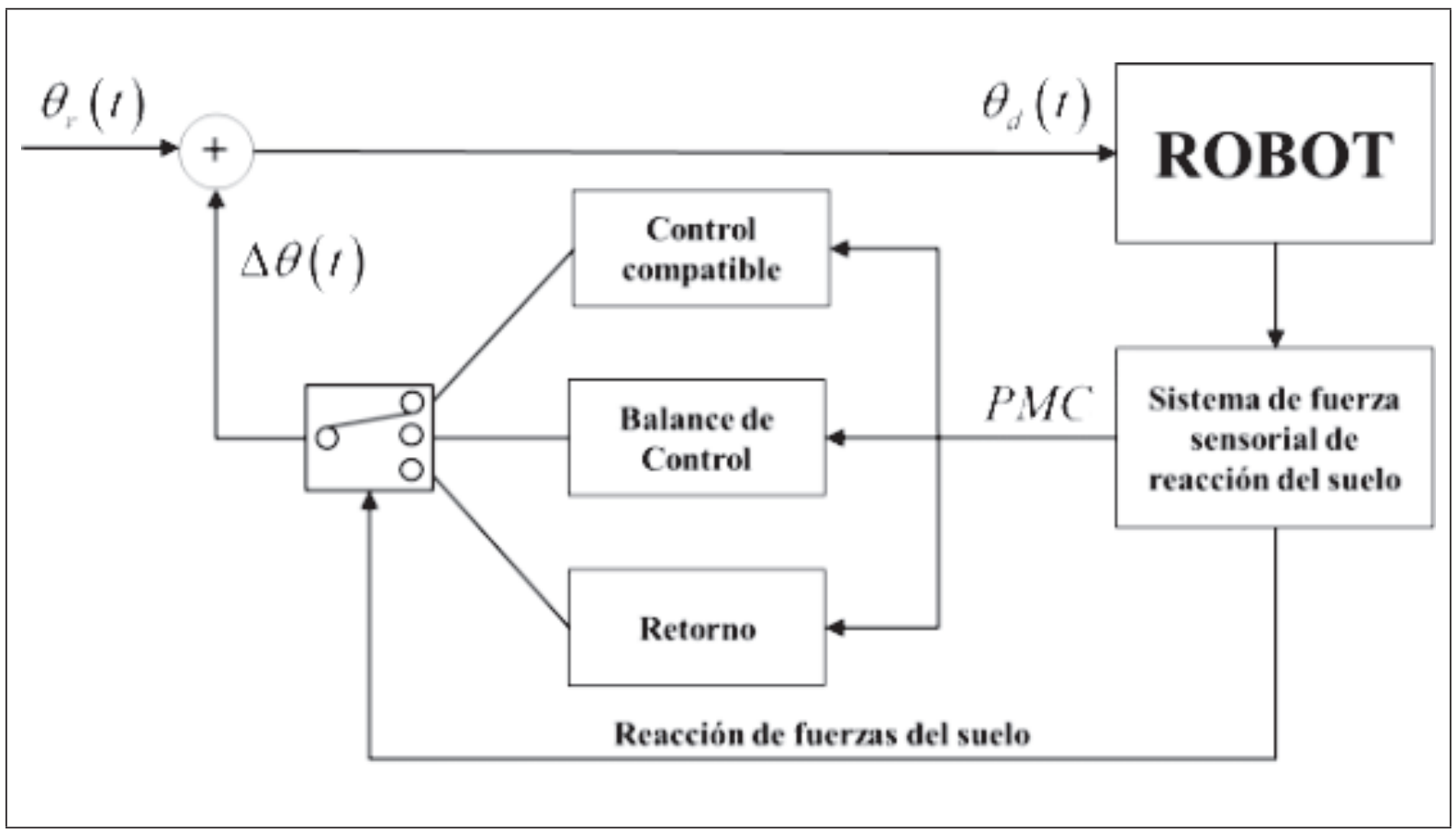

Figura 11. Estructura de control estabilizante para un robot de 14 grados de libertad

Fuente: Li et ál. (2010).

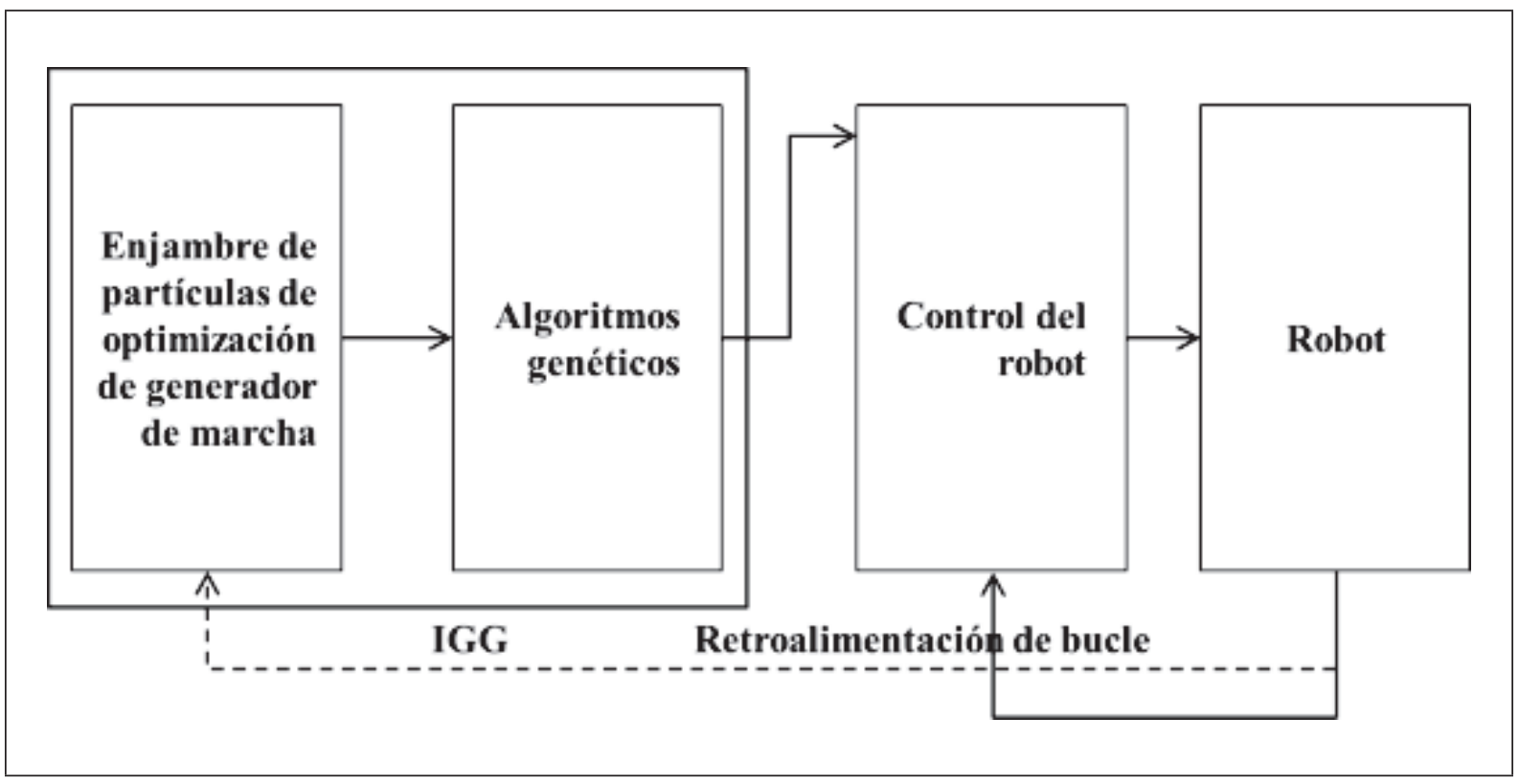

Figura 12. Flujo de control y compensación de torso superior

Fuente: Rokbani et ál. (2010). 
En muchos estudios las coordenadas del PMC se calculan utilizando el modelo del robot y la información del ángulo procedente del sensor de cada articulación. Sin embargo, en la investigación de Suwanratchatamanee, Matsumoto y Hashimoto (2010) se emplea una técnica que consiste en utilizar una retroalimentación táctil usando los sensores montados en las plantas de los pies del robot. El robot está controlado para mantener igual los tres valores de detección cuando el pie está apoyado. Cuando estos valores son similares, el CM también está en el centro del área de soporte. En otras palabras, esta técnica se puede emplear en el control del robot para mantener el CM constante en el centro del área de soporte en las fases de único y doble apoyo. El control de equilibrio es independiente del control de movimiento del robot; por lo tanto, solo se considera el control de movimiento para lograr la caminata cuando el control de balance está activo.

Kajita et ál. (2010) proponen la estabilización de la marcha para un robot con un mecanismo convencional en los pies. El controlador, como se muestra en la figura 13, se compone de tres capas: la de control de servo, la de control de fuerza y postura — que se encarga de la geometría del robot para controlar la postura corporal, los torques y las fuerzas de los pies-, y por último se encuentra la capa de control de PMC y CM del robot.

Van Heerden y Kawamura (2010) analizan las perturbaciones externas y la generación de trayectorias dinámicas en un robot bípedo. En el análisis se presenta un método para limitar las fuerzas de reacción del suelo. El método propuesto requiere como entradas la referencia de posición, los estados de las articulaciones y el dato del sensor de fuerza, como se observa en la figura 14. El control de posición basado en la observación del disturbio se utiliza para generar pares en el motor, los cuales robustamente harán que los ángulos de las articulaciones converjan a los valores de referencia. Para el control de fuerza, utilizan una perturbación del espacio de trabajo, a fin de observar y anular los efectos de las dinámicas de fricción, fuerzas externas, etc.

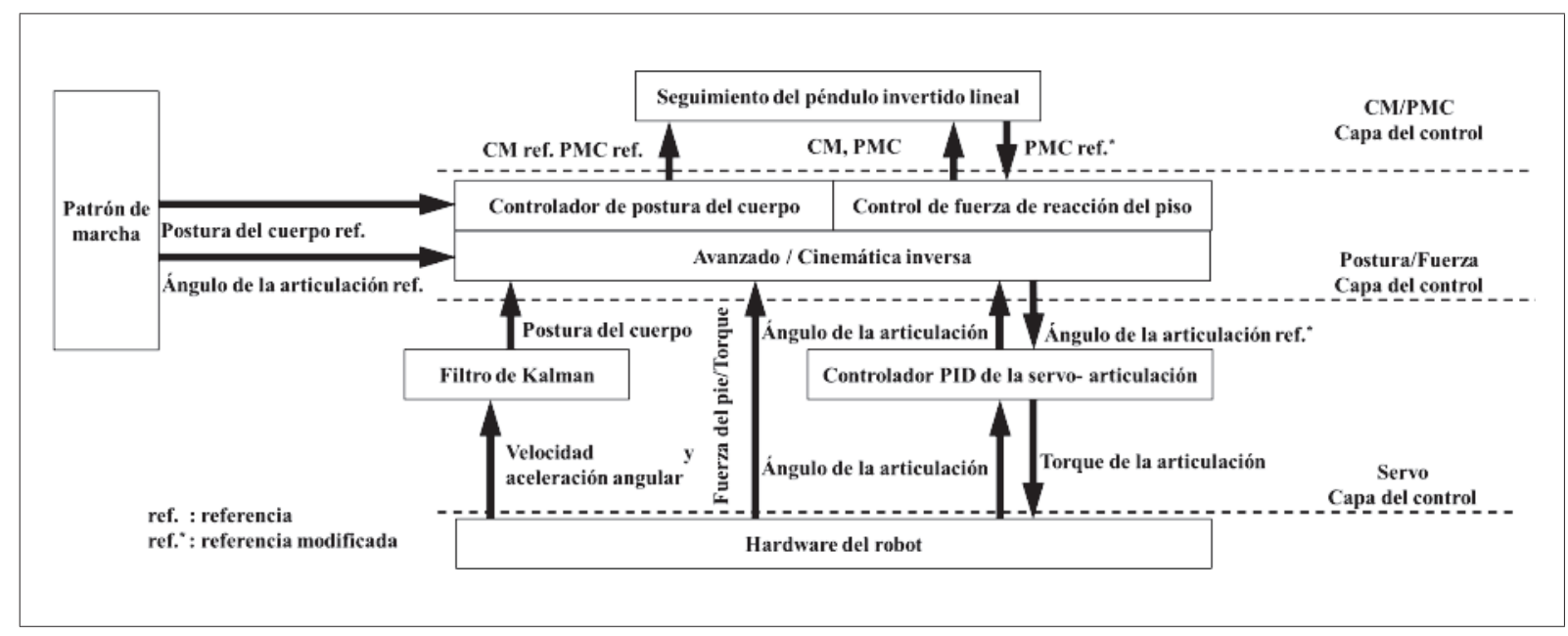

Figura 13. Estructura de control jerárquico

Fuente: Kajita et ál. (2010). 


\section{revisión}

Los péndulos invertidos han sido la base para la realización de la caminata bípeda, como se encuentra en la investigación de Aoyama, Sekiyama, Hasegawa y Fukuda (2010), donde los autores exponen la técnica de control autónomo dinámico pasivo utilizando un bípedo $3 \mathrm{D}$, caminado sobre un terreno irregular. A este efecto realizan el modelado como un péndulo invertido $3 \mathrm{D}$, equivalente a un sistema autónomo $2 \mathrm{D}$ mediante la aplicación del control autónomo de dinámica pasiva, basado en el punto de contacto de la base del robot y la restricción holonómica virtual como a las articulaciones del robot. Los autores afirman que el método del control autónomo dinámico pasivo tiene problemas en dividir la dinámica 3D cuando la dinámica de cada plano está fuertemente acoplada. Para resolver este problema se ha ampliado el enfoque del control autónomo dinámico pasivo sin dividir la dinámica $3 \mathrm{D}$ y, además, como se observa en la figura 15, se propone un método de estabilización y control de dirección de marcha para el robot Gorilla Robot II en un terreno irregular.

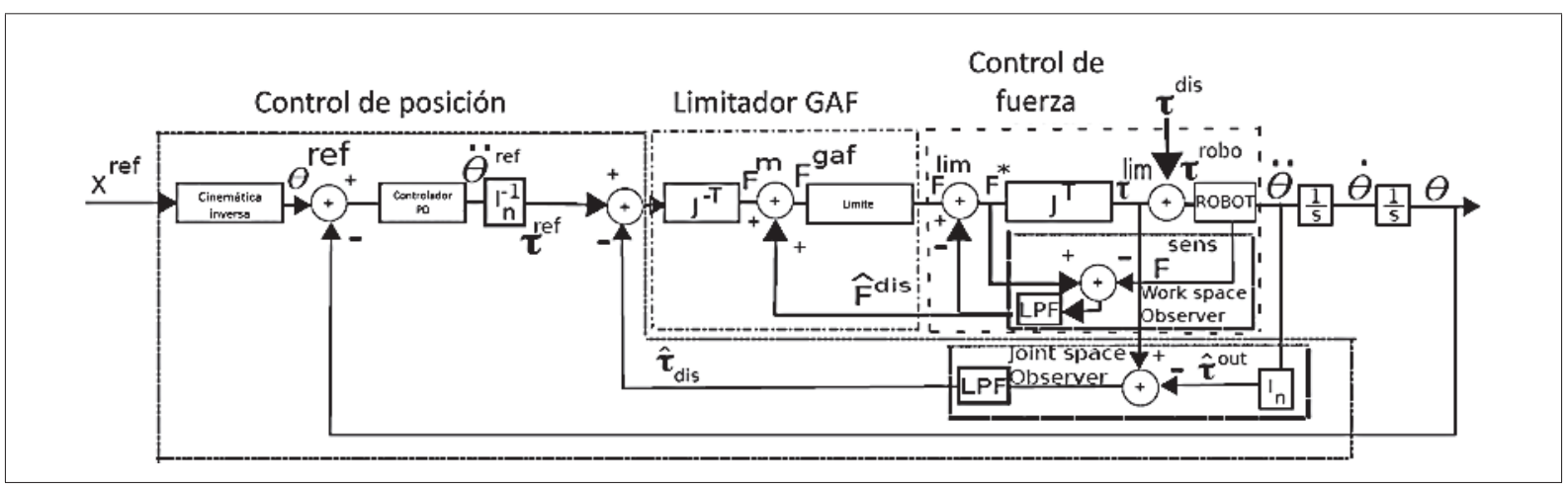

Figura 14. Control de posición con restricciones de fuerza

Fuente: Van Heerden y Kawamura (2010).

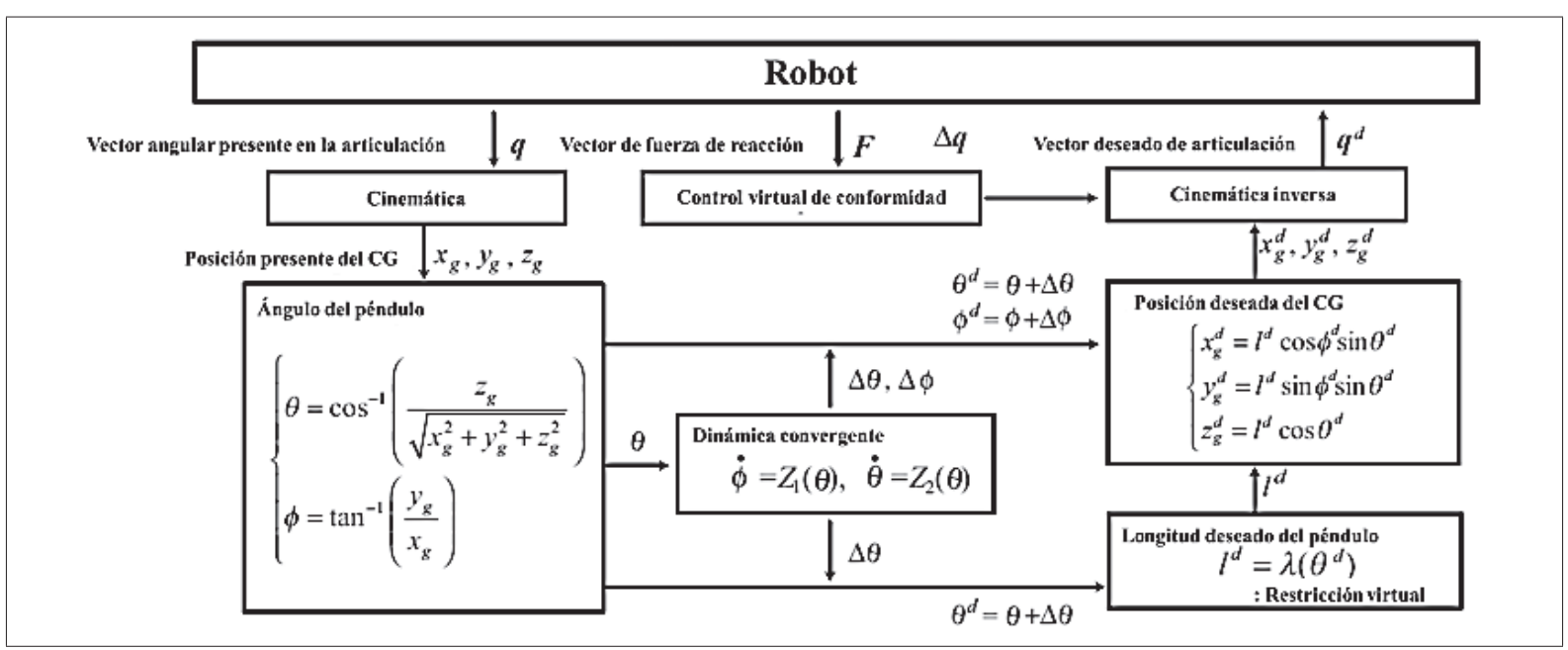

Figura 15. Arquitectura de control para el robot Gorilla

Fuente: Aoyama et ál. (2010). 
Yazdekhasti, Sheikholeslam y Ghayour (2010) proponen una ley de control invariante en el tiempo, donde se analiza una estrategia de control para regular la posición del PMC y las articulaciones del robot.

Benjamín y Atkeson (2010) presentan un método basado en modelos llamado control de fuerza para balance (equilibrio) dinámico, para la determinación de torques de todo el cuerpo basado en el $\mathrm{CM}$ deseado y las fuerzas de contacto para robots humanoides. La dinámica del CM se ve afectada directamente por el control de la fuerza de contacto para lograr un equilibrio estable. Esta idea se utiliza a fin de formular el control de fuerza para balance (equilibrio) dinámico, teniendo en cuenta toda la dinámica del robot para producir fuerzas de contacto deseadas.

Lim, Oh y Kim (2012) abordan el control del PMC mediante un control de balance. Para realizar el control de equilibrio se necesita la señal de posición del PMC solo como entrada, y a la salida se muestra un ángulo de compensación para las articulaciones afectadas. El rendimiento del sistema de control propuesto se simula y se comprueba en el robot humanoide MHR-1.

Ge, Li y Yang (2012) presentan una técnica de control adaptativo, usando funciones implícitas con soporte de vectores de regresión, en presencia de incertidumbres paramétricas y dinámicas funcio- nales. Con base en la síntesis de Lyapunov, desarrollan un control adaptativo desacoplado.

Hamed y Grizzle (2013) utilizan un control híbrido; de acuerdo con la simetría derecha-izquierda, se desarrollan desigualdades matriciales lineales y control óptimo robusto. En el primer nivel del controlador híbrido, el bucle de tiempo continuo emplea una clase general de las leyes de retroalimentación invariantes en el tiempo y no lineales para interactuar con el robot bípedo. Las leyes de retroalimentación en tiempo continuo son continuamente diferenciables y definidas por partes.

Las funciones de control de Lyapunov las utilizan Ames, Galloway y Grizzle (2012). Estos autores muestran cómo dichas funciones se pueden utilizar para estabilizar las órbitas periódicas de la dinámica híbrida cero de manera exponencial. Ames y Galloway (2013) también hacen uso de estas funciones. Presentan un método para la ampliación de la clase de controladores que estabilizan exponencialmente órbitas periódicas en sistemas híbridos. A partir de la dinámica cero, se presenta un enfoque del control a partir de las mencionadas funciones, que es exponencialmente estable. Esta técnica de control es probada en el robot Rabbit y en Mabel.

En la tabla 1 se encuentra información técnica sobre los documentos revisados en este estado de arte.

Tabla 1. Tabla de información sobre algunas especificaciones técnicas

\begin{tabular}{|c|c|c|c|c|c|c|c|c|c|c|}
\hline \multirow{2}{*}{ Ref. } & \multicolumn{2}{|c|}{ Pies } & \multirow{2}{*}{$\begin{array}{l}\text { Tam. } \\
(\mathrm{m})\end{array}$} & \multirow{2}{*}{ Super. } & \multicolumn{2}{|c|}{ Tіро } & \multirow{2}{*}{$\begin{array}{l}\text { Indicador de } \\
\text { medida }\end{array}$} & \multirow{2}{*}{$\begin{array}{l}\text { Estrategia de } \\
\text { control }\end{array}$} & \multirow{2}{*}{ Actuador } & \multirow{2}{*}{ GDL* } \\
\hline & Sí & No & & & Sim & Real & & & & \\
\hline $\begin{array}{l}\text { (Corpuz, Lafoteza, } \\
\text { Broas, y Ramos, 2009) }\end{array}$ & & & 0,74 & Plano & & & $\begin{array}{l}\text { Acelerómetro, } \\
\text { giróscopo }\end{array}$ & $\begin{array}{l}\text { Control de posi- } \\
\text { ción }\end{array}$ & Motor DC & 7 \\
\hline (Xin y Xiao, 2009) & & & -------- & & & & & $\begin{array}{l}\text { Control de posi- } \\
\text { ción }\end{array}$ & & 5 \\
\hline $\begin{array}{l}\text { (Ono, Sato y Ohnishi, } \\
\text { 2011) }\end{array}$ & & & 1,12 & Plano & & & $\begin{array}{l}\text { Sensor de } \\
\text { fuerza, aceleró- } \\
\text { metro }\end{array}$ & $\begin{array}{l}\text { Control PD, } \\
\text { Control de balance }\end{array}$ & & 10 \\
\hline
\end{tabular}


revisión

\begin{tabular}{|c|c|c|c|c|c|c|c|c|c|c|}
\hline \multirow{2}{*}{ Ref. } & \multicolumn{2}{|c|}{ Pies } & \multirow{2}{*}{$\begin{array}{l}\text { Tam. } \\
(\mathrm{m})\end{array}$} & \multirow{2}{*}{ Super. } & \multicolumn{2}{|c|}{ Tipo } & \multirow{2}{*}{$\begin{array}{l}\text { Indicador de } \\
\text { medida }\end{array}$} & \multirow{2}{*}{$\begin{array}{c}\text { Estrategia de } \\
\text { control }\end{array}$} & \multirow{2}{*}{ Actuador } & \multirow{2}{*}{ GDL* } \\
\hline & Sí & No & & & Sim & Real & & & & \\
\hline (Kim et ál., 2012) & & & 1,55 & Plano & & & $\begin{array}{l}\text { Sensor de } \\
\text { fuerza/torque }\end{array}$ & Control de par & $\begin{array}{l}\text { Motor DC } \\
\text { sin esco- } \\
\text { billas } \\
\end{array}$ & 6 \\
\hline (Roa y Ott, 2013) & & & 1,433 & $\begin{array}{l}\text { Plano } \\
\text { inclinado }\end{array}$ & & & $\begin{array}{l}\text { Sensor de } \\
\text { fuerza/torque }\end{array}$ & Control de balance & $\begin{array}{l}\text { Motor DC- } \\
\text { elástico }\end{array}$ & 6 \\
\hline (Zheng y Shen, 1990) & & & & $\begin{array}{l}\text { Plano } \\
\text { inclinado }\end{array}$ & & & $\begin{array}{l}\text { Sensor de po- } \\
\text { sición, sensor } \\
\text { de fuerza }\end{array}$ & $\begin{array}{l}\text { Esquema de con- } \\
\text { trol }\end{array}$ & Motor DC & 8 \\
\hline $\begin{array}{l}\text { (Kajita, Yamaura y } \\
\text { Kobayashi, 1992) }\end{array}$ & & & 0,37 & Plano & & & Potenciómetro & PD & Motor DC & 4 \\
\hline (Kajita y Tani, 1996) & & & 0,45 & Plano & & & \begin{tabular}{|l|} 
Potencióme- \\
tros, \\
Encoder de \\
pulso \\
\end{tabular} & $\begin{array}{l}\text { Realimentación } \\
\text { local, robot con } \\
\text { piernas ligeras }\end{array}$ & Motor DC & 6 \\
\hline (C. Shih, 1996) & & & 0,8 & Plano & & & |------------------- & Jerárquico & $\begin{array}{l}\text { Servomo- } \\
\text { tor DC }\end{array}$ & 7 \\
\hline (Goswami, 1999) & & & -------- & Plano & & & \begin{tabular}{|l|}
------------------- \\
\end{tabular} & Control del FRI & ------------- & ------ \\
\hline $\begin{array}{l}\text { ( Lim, Setiawan, y } \\
\text { Takanishi, 2001) }\end{array}$ & & & 1,88 & Plano & & & $\begin{array}{l}\text { Sensor de } \\
\text { fuerza/torque }\end{array}$ & $\begin{array}{l}\text { Híbrido posición/ } \\
\text { fuerza, impedancia }\end{array}$ & $\begin{array}{l}\text { Motor } \\
\text { lineal }\end{array}$ & 43 \\
\hline $\begin{array}{l}\text { (Pratt, Chew, Torres, } \\
\text { Dilworth y Pratt, 2001) }\end{array}$ & & & $\begin{array}{l}\text { ST: } 0,6 \\
\text { SF: } \\
0,9\end{array}$ & $\begin{array}{l}\text { Plano } \\
\text { inclinado }\end{array}$ & & & $\begin{array}{l}\text { Potencióme- } \\
\text { tros lineales }\end{array}$ & Modelo virtual & $\begin{array}{l}\text { Motor DC, } \\
\text { motor DC- } \\
\text { elástico }\end{array}$ & $\begin{array}{l}4 \\
6\end{array}$ \\
\hline (Rehbinder y Hu, 2001) & & & ---- & \begin{tabular}{|l|} 
Plano \\
inclinado
\end{tabular} & & & & $\begin{array}{l}\text { Arquitectura de } \\
\text { conmutación }\end{array}$ & ----- & ------ \\
\hline (Grizzle, 2002) & & & 1,43 & Plano & & & $\begin{array}{l}\text { Encoder ab- } \\
\text { soluto e incre- } \\
\text { mental }\end{array}$ & Feedback & Motor DC & 7 \\
\hline $\begin{array}{l}\text { (Chevallereau et ál., } \\
\text { 2003) }\end{array}$ & & & 1,43 & Plano & & & $\begin{array}{l}\text { Encoder ab- } \\
\text { soluto e incre- } \\
\text { mental } \\
\end{array}$ & $\begin{array}{l}\text { Limitaciones vir- } \\
\text { tuales, dinámica } \\
\text { híbrida cero }\end{array}$ & Motor DC & 7 \\
\hline $\begin{array}{l}\text { (Canudas-de-Wit, } \\
\text { 2004) }\end{array}$ & & & 1,43 & Plano & & & $\begin{array}{l}\text { Encoder ab- } \\
\text { soluto e incre- } \\
\text { mental } \\
\end{array}$ & $\begin{array}{l}\text { Limitaciones vir- } \\
\text { tuales }\end{array}$ & Motor DC & 7 \\
\hline (García, 2007) & & & 0,3 & Plano & & & $\begin{array}{l}\text { Sensor de } \\
\text { presión, } \\
\text { acelerómetros } \\
\end{array}$ & $\begin{array}{l}\text { Control de secuen- } \\
\text { cia, } \\
\text { control de par } \\
\end{array}$ & $\begin{array}{l}\text { Servomo- } \\
\text { tor DC }\end{array}$ & 2 \\
\hline (Kwon et ál., 2007) & & & 1,5 & Plano & & & $\begin{array}{l}\text { Sensor de } \\
\text { fuerza/torque, } \\
\text { Giróscopo, } \\
\text { micrófono, } \\
\text { cámara } \\
\end{array}$ & $\begin{array}{l}\text { Control de impe- } \\
\text { dancia }\end{array}$ & Motor DC & 35 \\
\hline $\begin{array}{l}\text { (Lin, Chen y Chen, } \\
\text { 2007) }\end{array}$ & & & --- & Plano & & & --------- & Control híbrido & -- & 6 \\
\hline $\begin{array}{l}\text { (C. L. Shih, Grizzle y } \\
\text { Chevallereau, 2007) }\end{array}$ & & & & Plano & & & ------- & $\begin{array}{l}\text { Control feedback, } \\
\text { Control basado en } \\
\text { eventos }\end{array}$ & & 6 \\
\hline $\begin{array}{l}\text { (Honda Motor Co. Ltd, } \\
\text { 2007) }\end{array}$ & & & 1,2 & Plano & & & $\begin{array}{l}\text { Giróscopo, } \\
\text { sensor de } \\
\text { fuerza, cámara, } \\
\text { antena, acele- } \\
\text { rómetro } \\
\end{array}$ & $\begin{array}{l}\text { Control de pre- } \\
\text { dicción de movi- } \\
\text { miento }\end{array}$ & $\begin{array}{l}\text { Servomo- } \\
\text { tor DC, } \\
\text { reductor } \\
\text { de veloci- } \\
\text { dad } \\
\end{array}$ & 12 \\
\hline (Anybots, 2007) & & & |------- & Plano & & & \begin{tabular}{|l|}
-------------- \\
\end{tabular} & Control del CM & ----------- & ------ \\
\hline
\end{tabular}

156 Tecnura | Vol. 19 | No. 43 | enero - marzo de 2015 


\begin{tabular}{|c|c|c|c|c|c|c|c|c|c|c|}
\hline \multirow{2}{*}{ Ref. } & \multicolumn{2}{|c|}{ Pies } & \multirow{2}{*}{$\begin{array}{l}\text { Tam. } \\
\text { (m) }\end{array}$} & \multirow{2}{*}{ Super. } & \multicolumn{2}{|c|}{ Tipo } & \multirow{2}{*}{$\begin{array}{l}\text { Indicador de } \\
\text { medida }\end{array}$} & \multirow{2}{*}{$\begin{array}{l}\text { Estrategia de } \\
\text { control }\end{array}$} & \multirow{2}{*}{ Actuador } & \multirow{2}{*}{ GDL* } \\
\hline & Sí & No & & & Sim & Real & & & & \\
\hline $\begin{array}{l}\text { (Jessy W. Grizzle, } \\
\text { 2008) }\end{array}$ & & & 2,25 & $\begin{array}{l}\text { Plano, } \\
\text { escalera }\end{array}$ & & & $\begin{array}{l}\text { Sensor de con- } \\
\text { tacto, sensor } \\
\text { de ángulo }\end{array}$ & $\begin{array}{l}\text { Control híbrido } \\
\text { cero }\end{array}$ & $\begin{array}{l}\text { Motor DC } \\
\text { sin esco- } \\
\text { billas }\end{array}$ & 7 \\
\hline $\begin{array}{l}\text { (Matt, Shane, Allison y } \\
\text { Philip, 2010) }\end{array}$ & & & 2,25 & $\begin{array}{l}\text { Plano, } \\
\text { escalera }\end{array}$ & & & $\begin{array}{l}\text { Sensor de con- } \\
\text { tacto, sensor } \\
\text { de ángulo }\end{array}$ & $\begin{array}{l}\text { Control híbrido } \\
\text { cero }\end{array}$ & $\begin{array}{l}\text { Motor DC } \\
\text { sin esco- } \\
\text { billas }\end{array}$ & 7 \\
\hline (Aclan y Ramos, 2009) & & & 1,31 & Plano & & & $\begin{array}{l}\text { Potenciómetro } \\
\text { de precisión, } \\
\text { giróscopo, } \\
\text { acelerómetro }\end{array}$ & $\begin{array}{l}\text { Control multivaria- } \\
\text { ble PID }\end{array}$ & Motor DC & 6 \\
\hline $\begin{array}{l}\text { (Matsumoto y } \\
\text { Kawamura, 2010) }\end{array}$ & & & 1,22 & Plano & & & $\begin{array}{l}\text { Giróscopo, } \\
\text { acelerómetro, } \\
\text { sensor de fuer- } \\
\text { za/torque }\end{array}$ & $\begin{array}{l}\text { Control de direc- } \\
\text { ción robusto }\end{array}$ & $\begin{array}{l}\text { Servomo- } \\
\text { tor DC }\end{array}$ & 13 \\
\hline $\begin{array}{l}\text { (Ferreira, Crisóstomo y } \\
\text { Coimbra, 2010) }\end{array}$ & & & 0,5 & \begin{tabular}{|l|} 
Esca- \\
leras, \\
plano, \\
inclinado
\end{tabular} & & & $\begin{array}{l}\text { Sensor de } \\
\text { fuerza }\end{array}$ & $\begin{array}{l}\text { Control de inteli- } \\
\text { gencia artificial }\end{array}$ & $\begin{array}{l}\text { Servomo- } \\
\text { tor DC }\end{array}$ & 6 \\
\hline $\begin{array}{l}\text { (Parsa y Farrokhi, } \\
\text { 2010) }\end{array}$ & & & 1,32 & Plano & & & ----------------- & $\begin{array}{l}\text { Modelo de control } \\
\text { predictivo no lineal }\end{array}$ & ----------- & 5 \\
\hline $\begin{array}{l}\text { (Aghabalaie, Hos- } \\
\text { seinzadeh, Talebi y } \\
\text { Shafiee, 2010) }\end{array}$ & & & 1,12 & Plano & & & ---------------- & $\begin{array}{l}\text { Control robusto no } \\
\text { lineal }\end{array}$ & |----------- & 5 \\
\hline (G. Li et ál., 2010) & & & 0,27 & Plano & & & $\begin{array}{l}\text { Sensor de } \\
\text { fuerza }\end{array}$ & $\begin{array}{l}\text { Control de estabi- } \\
\text { lidad }\end{array}$ & $\begin{array}{l}\text { Servomo- } \\
\text { tor DC }\end{array}$ & 7 \\
\hline $\begin{array}{l}\text { (Rokbani, Benbousaa- } \\
\text { da, Ammar y Alimi, } \\
\text { 2010) }\end{array}$ & & & -------- & ------- & & & $\begin{array}{l}\text { Sensor infrarro- } \\
\text { jo, micrófono }\end{array}$ & Control de CM & $\begin{array}{l}\text { Servomo- } \\
\text { tor DC }\end{array}$ & 7 \\
\hline $\begin{array}{l}\text { (Suwanratchatamanee, } \\
\text { Matsumoto y Hashi- } \\
\text { moto, 2010) }\end{array}$ & & & 0,34 & $\begin{array}{l}\text { Plano, } \\
\text { inclinado }\end{array}$ & & & Sensor táctil & Control distribuido & $\begin{array}{l}\text { Servomo- } \\
\text { tor DC }\end{array}$ & 17 \\
\hline $\begin{array}{l}\text { (Shuuji Kajita et ál., } \\
\text { 2010) }\end{array}$ & & & 1,58 & $\begin{array}{l}\text { Plano, } \\
\text { inclinado }\end{array}$ & & & $\begin{array}{l}\text { Sensor de fuer- } \\
\text { za, giróscopo, } \\
\text { acelerómetro, } \\
\text { sensor de po- } \\
\text { sición, Encoder } \\
\text { incremental }\end{array}$ & $\begin{array}{l}\text { Control de postu- } \\
\text { ra/fuerza, PID }\end{array}$ & $\begin{array}{l}\text { Servomo- } \\
\text { tor DC }\end{array}$ & 42 \\
\hline $\begin{array}{l}\text { (Chevallereau, Grizzle y } \\
\text { Shih, 2009) }\end{array}$ & & & 1,43 & Plano & & & $\begin{array}{l}\text { Encoder ab- } \\
\text { soluto e incre- } \\
\text { mental }\end{array}$ & $\begin{array}{l}\text { Control de reali- } \\
\text { mentación inva- } \\
\text { riante en el tiempo }\end{array}$ & Motor DC & 7 \\
\hline $\begin{array}{l}\text { (Van Heerden y } \\
\text { Kawamura, 2010) }\end{array}$ & & & 1,216 & Plano & & & $\begin{array}{l}\text { Sensor de fuer- } \\
\text { za, giróscopo, } \\
\text { Acelerómetro }\end{array}$ & $\begin{array}{l}\text { Control basado en } \\
\text { la observación del } \\
\text { disturbio }\end{array}$ & $\begin{array}{l}\text { Servomo- } \\
\text { tor DC }\end{array}$ & 6 \\
\hline $\begin{array}{l}\text { (Aoyama, Sekiyama, } \\
\text { Hasegawa y Fukuda, } \\
2010 \text { ) }\end{array}$ & & & 0,905 & Plano & & & $\begin{array}{l}\text { Sensor de fuer- } \\
\text { za, giróscopo, } \\
\text { Encoder }\end{array}$ & $\begin{array}{l}\text { Control autónomo } \\
\text { dinámico pasivo }\end{array}$ & $\begin{array}{l}\text { Servomo- } \\
\text { tor AC }\end{array}$ & 10 \\
\hline $\begin{array}{l}\text { (Yazdekhasti, Sheik- } \\
\text { holeslam y Ghayour, } \\
\text { 2010) }\end{array}$ & & & 1,685 & --------- & & & ------------------- & Control de PMC & ---------- & 7 \\
\hline (Li, Su y Liu, 2012) & & & 0,22 & Plano & & & $\begin{array}{l}\text { Sensor de } \\
\text { fuerza/torque, } \\
\text { acelerómetro }\end{array}$ & $\begin{array}{l}\text { Control de fuerza } \\
\text { de balance diná- } \\
\text { mico }\end{array}$ & Hidráulico & 33 \\
\hline
\end{tabular}




\section{revisión}

\begin{tabular}{|c|c|c|c|c|c|c|c|c|c|c|}
\hline \multirow{2}{*}{ Ref. } & \multicolumn{2}{|c|}{ Pies } & \multirow{2}{*}{$\begin{array}{l}\text { Tam. } \\
(\mathrm{m})\end{array}$} & \multirow{2}{*}{ Super. } & \multicolumn{2}{|c|}{ Tipo } & \multirow{2}{*}{$\begin{array}{c}\text { Indicador de } \\
\text { medida }\end{array}$} & \multirow{2}{*}{$\begin{array}{c}\text { Estrategia de } \\
\text { control }\end{array}$} & \multirow{2}{*}{ Actuador } & \multirow{2}{*}{ GDL* } \\
\hline & Sí & No & & & Sim & Real & & & & \\
\hline $\begin{array}{l}\text { (S. Lim, Oh y Kim, } \\
\text { 2012) }\end{array}$ & & & 1,45 & Plano & & & $\begin{array}{l}\text { Sensor de } \\
\text { fuerza, } \\
\text { giróscopo, } \\
\text { sensor infra- } \\
\text { rrojo }\end{array}$ & $\begin{array}{l}\text { Control de posi- } \\
\text { ción del PMC }\end{array}$ & $\begin{array}{l}\text { Servomo- } \\
\text { tor DC }\end{array}$ & 24 \\
\hline (Ge, Li y Yang, 2012) & & & 1,1228 & Plano & & & --- & $\begin{array}{l}\text { Control adaptativo } \\
\text { predictivo }\end{array}$ & -------- & 7 \\
\hline $\begin{array}{l}\text { (Hamed y Grizzle, } \\
\text { 2013) }\end{array}$ & & & -------- & Plano & & & $\begin{array}{l}\text { Encoder ab- } \\
\text { soluto e incre- } \\
\text { mental } \\
\end{array}$ & $\begin{array}{l}\text { Control híbrido } \\
\text { (control óptimo) }\end{array}$ & $\begin{array}{l}\text { Motor- } \\
\text { elástico }\end{array}$ & 13 \\
\hline $\begin{array}{l}\text { (Ames, Galloway y } \\
\text { Grizzle, 2012) }\end{array}$ & & & & Plano & & & ------ & $\begin{array}{l}\text { Control de función } \\
\text { de Lyapunov }\end{array}$ & & |----- \\
\hline $\begin{array}{l}\text { (Ames y Galloway, } \\
\text { 2013) }\end{array}$ & & & $\begin{array}{l}\text { R: } 2,25 \\
\text { M: } \\
2,25\end{array}$ & $\begin{array}{l}\text { Plano, } \\
\text { escalera }\end{array}$ & & & $\begin{array}{l}\text { Sensor de con- } \\
\text { tacto, sensor } \\
\text { de ángulo }\end{array}$ & $\begin{array}{l}\text { Control de función } \\
\text { de Lyapunov }\end{array}$ & $\begin{array}{l}\text { Motor DC } \\
\text { sin esco- } \\
\text { billas }\end{array}$ & 7 \\
\hline
\end{tabular}

* GDL: Grados de libertad

Fuente: elaboración propia.

\section{CONCLUSIONES}

El estado actual de las investigaciones en robots bípedos muestra que la locomoción bípeda estable no está totalmente resuelta y que en el mercado no hay un gran número de robots con tamaño de un humano, debido a que el problema de locomoción bípeda aumenta con la altura y el peso del robot.

Obtener una marcha correcta y rápida en un robot bípedo implica transformar en cada instante la posición de cada pie en una posición de articulación, es decir, generar trayectorias de marcha en espacio y tiempo. Para ello se necesita hacer el cálculo de las cinemáticas directa e inversa del modelo diseñado, lo cual requiere un cálculo computacional.

Como un índice de desempeño en la estabilidad de un robot bípedo, el PMC es el criterio más utilizado e implementado en esta área. Todas estas técnicas de control para el equilibrio y la variedad de metodologías para la obtención de una marcha natural, fácilmente se pueden aplicar a sistemas de exoesqueletos para lograr la rehabilitación de la marcha y el equilibrio en humanos.

Finalmente, con la clasificación obtenida para estrategias de control aplicadas a los robots bípedos, se logró evidenciar que el control de equilibrio dinámico y la generación de patrones de marcha son temas de interés para estudios posteriores, porque el objetivo general para la robótica bípeda es obtener movimientos suaves y naturales como el ser humano interactuando en diferentes ambientes.

\section{FINANCIAMIENTO}

Esta investigación forma parte del proyecto Marcha asistida con un exoesqueleto, de la Universidad del Valle y Colciencias. 


\section{REFERENCIAS}

Abdallah, M. y Goswami, A. (2005). A Biomechanically Motivated Two-Phase Strategy for Biped Upright Balance Control. En Proceedings of the 2005 IEEE International Conference on Robotics and Automation (vol. 2, pp. 1996-2001). IEEE. doi:10.1109/ ROBOT.2005.1570406.

Aclan, M. C. y Ramos, M. C. (2009). Bipedal Robot Locomotion Using Multivariable Control. En TENCON 2009-2009 IEEE Region 10 Conference (pp. 1-6). IEEE. doi:10.1109/TENCON.2009.5396036.

Aghabalaie, P., Hosseinzadeh, M., Talebi, H. A. y Shafiee, M. (2010). Nonlinear Robust Control of a Biped Robot. En 2010 IEEE International Symposium on Industrial Electronics (pp. 1907-1912). IEEE. doi:10.1109/ISIE.2010.5637535.

Ames, A. D., Galloway, K. y Grizzle, J. W. (2012). Control Lyapunov Functions and Hybrid Zero Dynamics. 2012 IEEE 51st IEEE Conference on Decision and Control (CDC) (pp. 6837-6842). doi:10.1109/ CDC.2012.6426229.

Ames, A. y Galloway, K. (2013). Rapidly Exponentially Stabilizing Control Lyapunov Functions and Hybrid Zero Dynamics. IEEE Transactions on Automatic Control, 59 (4), 1-15.

Anybots, I. (2007). Anybots. Recuperado el 10 de agosto del 2011, dehttps://www.anybots.com

Aoyama, T., Sekiyama, K., Hasegawa, Y. y Fukuda, T. (2010). 3-D Biped Walking over Rough Terrain Based on the Assumption of Point-Contact. En 2010 IEEE/RSJ In- ternational Conference on Intelligent Robots and Systems (pp. 3163-3168). IEEE. doi:10.1109/IROS.2010.5650891

Canudas-de-Wit, C. (2004). On the Concept of Virtual Constraints as a Tool for Walking Robot Control and Balancing. Annual Reviews in Control, 28 (2), 157-166. doi:10.1016/j.arcontrol.2004.03.002.

Chevallereau, C., Abba, G., Aoustin, Y., Plestan, F., Westervelt, E. R., Canudas-de-Wit, C. y Grizzle, J. W. (2003). RABBIT: A Testbed for Advanced Control Theory. IEEE Control Systems Magazine, 23 (5), 57-79. doi:10.1109/MCS.2003.1234651.

Chevallereau, C., Grizzle, J. W. y Shih, C. L. (2009). Asymptotically Stable Walking of a Five-Link Underactuated 3-D Bipedal Robot. IEEE Transactions on Robotics, 25 (1), 37-50. doi:10.1109/ TRO.2008.2010366.

Corpuz, F. J. O., Lafoteza, B. C. Y., Broas, R. A. L. y Ramos, M. (2009). Design and Implementation of a Closed-Loop Static Balance System for the YICAL Leg 2 Biped. En TENCON 2009-2009 IEEE Region 10 Conference (pp. 1-6). Singapur: IEEE. doi:10.1109/TENCON.2009.5395815.

Fernández Iglesias, S. (2009). Locomoción bípeda del robot humanoide Nao. Universidad de Cataluña.

Ferreira, J. P., Crisóstomo, M. M. y Coimbra, A. P. (2010). SVR Sagittal Balance of a Biped Robot Controlling the Torso and Ankle Joint Angles. En 2010 IEEE International Conference on Industrial Engineering and 
Engineering Management (pp. 1931-1936). IEEE. doi:10.1109/IEEM.2010.5674629

García, I. (2007). Introduction to Dynamic Balance for Humanoid Robots, the Key of Biped Locomotion. Communications (pp. 1-4). Málaga.

Ge, S. S., Li, Z. y Yang, H. (2012). Data Driven Adaptive Predictive Control for Holonomic Constrained Under-Actuated Biped Robots. IEEE Transactions on Control Systems Technology, 20 (3), 787-795. doi:10.1109/TCST.2011.2145378.

Grizzle, J. W. (2002). Rabbit Robot Biped. Recuperado el $1^{\circ}$ de septiembre del 2011, de http://web.eecs.umich.edu/ grizzle/papers/RABBITExperiments.html.

Grizzle, J. W. (2008). Mabel. Recuperado el $1^{\circ}$ de septiembre del 2008, de http://web.eecs. umich.edu/ grizzle/papers/MABEL.html.

Guihard, M. y Gorce, P. (2002). Dynamic Control of Bipeds Using Ankle and Hip Strategies. En IEEE/RSJ International Conference on Intelligent Robots and System (vol. 3, pp. 2587-2592). IEEE. doi:10.1109/ IRDS.2002.1041660.

Guzmán Valdivia, C. H. (2010). Construcción de un robot bípedo basado en caminado dinámico. Centro Nacional de Investigación y Desarrollo Tecnológico.

Hamed, K. y Grizzle, J. W. (2013). Event-Based Stabilization of Periodic Orbits for Underactuated 3D Bipedal Robots with LeftRight Symmetry. IEEE Transactions on Robotics, en revisión, 1-16.

Honda Motor (2007). Robot Asimo. Recuperado de http://asimo.honda.com/downloads/pdf/ asimo-technical-information.pdf.
Kajita, S., Morisawa, M., Miura, K., Nakaoka, S., Harada, K., Kaneko, K, Yokoi, K. et ál. (2010). Biped Walking Stabilization Based on Linear Inverted Pendulum Tracking. En 2010 IEEE/RSJ International Conference on Intelligent Robots and Systems (pp. 4489-4496). IEEE. doi:10.1109/ IROS.2010.5651082

Kim, J., Kwak, H., Lee, H., Seo, K., Lim, B., Lee, M., Roh, K. et ál. (2012). Balancing Control of a Biped Robot. En 2012 IEEE International Conference on Systems, Man, and Cybernetics (SMC) (pp. 2756-2761). IEEE. doi:10.1109/ICSMC.2012.6378165.

Kwon, W., Kim, H. K., Park, J. K., Roh, C. H., Lee, J., Park, J., Roh, K. et ál. (2007). Biped Humanoid Robot Mahru III. En 2007 7th IEEE-RAS International Conference on Humanoid Robots (pp. 583-588). IEEE. doi:10.1109/ICHR.2007.4813930.

Li, G., Huang, Q., Xu, Q., Li, G., Li, J. y Li, M. (2010). Design of a Small Biped Mechanism with 7 DOFs Legs and Double Spherical Hip Joint. En $20108^{\text {th }}$ World Congress on Intelligent Control and Automation (pp. 264-269). IEEE. doi:10.1109/WCICA.2010.5554999.

Li, T., Su, Y. y Liu, S.-H. (2012). Dynamic Balance Control for Biped Robot Walking Using Sensor Fusion, Kalman Filter, and Fuzzy Logic. IEEE Transactions on Robotics, 59 (11), 4394-4408. doi:10.1109/ TIE.2011.2175671.

Li, Z. y Ge, S. S. (2012). Adaptive Robust Controls of Biped Robots. IET Control Theoryy Applications, 7 (2), 161-175. doi:10.1049/ iet-cta.2012.0066.

Lim, H., Setiawan, S. A. y Takanishi, A. (2001). Balance and Impedance Control for Biped 
Humanoid Robot Locomotion. En Proceedings 2001 IEEE/RSJ International Conference on Intelligent Robots and Systems. Expanding the Societal Role of Robotics in the Next Millennium (Cat. No.01CH37180) (vol. 1, pp. 494-499). IEEE. doi:10.1109/ IROS.2001.973405.

Lim, S., Oh, S. N. y Kim, K. I. (2012). Balance Control for Biped Walking Robots Usingonly Zero-Moment-Point Position Signal. Electronics Letters, 48 (1), 19. doi:10.1049/ el.2011.3091.

Lin, C. M., Chen, L. Y. y Chen, C. H. (2007). RCMAC hybrid control for MIMO Uncertain Nonlinear Systems Using Sliding-Mode Technology. IEEE Transactions on Neural Network, 18 (3), 708-20. doi:10.1109/ TNN.2007.891198.

Liu, G., Li, M., Guo, W. y Cai, H. (2012). Control of a Biped Walking with Dynamic Balance. 2012 IEEE International Conference on Mechatronics and Automation (pp. 261267). doi:10.1109/ICMA.2012.6282852.

Matsumoto, K. y Kawamura, A. (2010). The Direction Control of a Biped Robot Using Gyro Sensor Feedback. En 2010 11th IEEE International Workshop on Advanced Motion Control (AMC) (pp. 137-142). IEEE. doi:10.1109/AMC.2010.5464012.

Matt, R., Shane, B., Allison, L. y Philip, V. (2010). MABEL Robotic Foot Design Final Report ME 450 Fall 2010.

Ono, H., Sato, T. y Ohnishi, K. (2011). Balance Recovery of Ankle Strategy: Using Knee Joint for Biped Robot. Access Spaces, 1, 236-241. doi:10.1109/ISAS.2011.5960955.

Pardos Gotor, J. M. (2005). Algoritmos de geometría diferencial para la locomoción y navegación bipedas de robots humanoides aplicación al robot RHO director. Madrid: Universidad Carlos III de Madrid.

Parsa, M. y Farrokhi, M. (2010). Robust Nonlinear Model Predictive Trajectory Free Control of Biped Robots Based on Nonlinear Disturbance Observer. En $201018^{\text {th }}$ Iranian Conference on Electrical Engineering (pp. 617-622). IEEE. doi:10.1109/IRANIANCEE.2010.5506996.

Pratt, J. E., Chew, C. M., Torres, A., Dilworth, P. y Pratt, G. (2001). Virtual Model Control: An Intuitive Approach for Bipedal Locomotion. The International Journal of Robotics Research, 20 (2), 129-143. doi:10.1177/02783640122067309.

Rehbinder, H. y Hu, X. (2001). Drift-Free Attitude Estimation for Accelerated Rigid Bodies. En Proceedings 2001 ICRA. IEEE International Conference on Robotics and Automation (Cat. No.01CH37164) (vol. 4, pp. 4244-4249). IEEE. doi:10.1109/ROBOT.2001.933281

Roa, M. y Ott, C. (2013). Balance and Posture Control for Biped Robots. En Multibody System Dynamics, Robotics and Control (pp. 129-143). doi:10.1007/978-3-70911289-2 8

Rokbani, N., Benbousaada, E., Ammar, B. y Alimi, A. M. (2010). Biped Robot Control Using Particle Swarm Optimization. En 2010 IEEE International Conference on Systems, Man and Cybernetics (pp. 506-512). IEEE. doi:10.1109/ICSMC.2010.5642027

Santana Hernández, J. L. (2006). Control del equilibrio en el proceso de caminado de un robot bipedo. Universidad de Guadalajara. 
Sheng-jun, P., Hai-tao, S., Tao, S. y Hong-xu, M. (2009). A novel Stability Criterion for Underactuated Biped Robot. En 2009 IEEE International Conference on Robotics and Biomimetics (ROBIO) (pp. 1912-1917). IEEE. doi:10.1109/ROBIO.2009.5420550.

Shih, C. L., Grizzle, J. W. y Chevallereau, C. (2007). Asymptotically Stable Walking of a Simple Underactuated 3D Bipedal Robot. IECON 2007-33 rd Annual Conference of the IEEE Industrial Electronics Society (vol. 1, pp. 2766-2771). doi:10.1109/ IECON.2007.4460177.

Stephens, B. J. y Atkeson, C. G. (2010). Dynamic Balance Force Control for Compliant $\mathrm{Hu}-$ manoid Robots. En 2010 IEEE/RSJ International Conference on Intelligent Robots and Systems (pp. 1248-1255). IEEE. doi:10.1109/IROS.2010.5648837.

Suwanratchatamanee, K., Matsumoto, M. y Hashimoto, S. (2010). Walking on the Slopes with Tactile Sensing System for Humanoid Robot. En Control Automation and Systems (ICCAS), 2010 International Conference on (pp. 350-355). IEEE.

Van Heerden, K. y Kawamura, A. (2010). An Investigation on Robust Biped Walking Using Compliant Force Control and a Online Walking Pattern Generator. En IECON 2010-36 ${ }^{\text {th }}$ Annual Conference on IEEE Industrial Electronics Society (pp. 1453-1458). IEEE. doi:10.1109/ IECON.2010.5675470.

Xin, R. y Xiao, N. (2009). A Novel Compound Biped Locomotion Algorithm for Humanoid Robots to Realize Biped Walking. Journal of Control Theory and Applications, 7 (1), 23-28. doi:10.1007/s11768009-7153-Z

Yazdekhasti, S., Sheikholeslam, F. y Ghayour, M. (2010). Stability Analysis of Biped Robot with Direct Control of Zero Moment Point. En Computer and Automation Engineering ICCAE 2010 The $2^{\text {nd }}$ International Conference (pp. 528-532). IEEE. doi:10.1109/ICCAE.2010.5451643.

Yeoun-Jae, K., Joon-Yong, L. y Ju-Jang, L. (2012). A Balance Control Strategy of a Walking Biped Robot in an Externally Applied Force. Information and Automation, 572-577.

Zermeño Sobrino, P. M. (2006). Elementos con rotación atrás. En Manual para el entrenador de gimnasia de trampolín Nivel 2 (pp. 25-39). México: Dirección de la Escuela Nacional de Entrenadores Deportivos. 